\title{
Two-beam accelerator based on a Cherenkov wake amplified by an optical active medium
}

\author{
Naama Cohen๑* and Levi Schächter \\ Technion-Israel Institute of Technology Haifa 32000, Israel
}

(Received 1 September 2020; accepted 2 November 2020; published 24 November 2020)

\begin{abstract}
We develop the basic scaling laws for the design of a proof of principle two-beam accelerator based on a solid-state active medium (TBA-AM). The essence of the paradigm is to rely on a low-charge trigger bunch which will generate a weak intensity Cherenkov wake in a dielectric extraction structure. The wake consists of a discrete spectrum of eigenfrequencies, one of which is amplified by the active dielectric material. The exponential growth of the wake, behind the trigger bunch, continues as long as virtually all the energy from the medium is depleted. At this point, the electromagnetic field reaches saturation and thus its maximum value. Beyond this peak gradient, a trailing bunch may be placed and thus accelerated. Evidently, the major difference between the proposed paradigm and the existing ones is the energy source: in existing schemes the energy is stored in the driving bunch, whereas in ours, the required energy is stored in the active medium. Special attention is paid to three quantities which characterize the interacting mode: its initial amplitude generated by the trigger bunch, its exponential growth, and its saturation level. The latter indicates that accelerating gradients in excess of $1 \mathrm{GV} / \mathrm{m}$ are feasible in a less than $10 \mathrm{~cm}$ long structure.
\end{abstract}

DOI: 10.1103/PhysRevAccelBeams.23.111303

\section{INTRODUCTION}

Linear accelerators are designed to deliver high energy beams of charged subatomic particles, for various purposes. Their applications range from the few meter long linacs which produce electrons at the $\mathrm{MeV}$ scale for radiotherapy [1], to the $31.5 \mathrm{~km}$ long International Linear Collider (ILC) designed to smash electrons and positrons of $500 \mathrm{GeV}$ [2] for high energy physics purposes. All existing linear accelerators are driven by rf sources [3] however intensive efforts are invested in plasma based wakefield where the latter is generated by either optical or electron intense pulses $[4,5]$ however, these are beyond the scope of the present study.

Acceleration of electrons at gradients higher than those achieved in the state-of-art technologies, while maintaining the accelerating structures compact, are two key factors for next-generation linacs. For that end, the two-beam acceleration (TBA) is a promising technique in which energy from a typically high-charge medium-energy drive bunch is transferred to a low-current, high-energy trailing electron bunch. This energy transfer can be achieved when the drive bunch generates a wakefield, while the trailing bunch that

\footnotetext{
*snaamac@campus.technion.ac.il
}

Published by the American Physical Society under the terms of the Creative Commons Attribution 4.0 International license. Further distribution of this work must maintain attribution to the author(s) and the published article's title, journal citation, and DOI. follows is accelerated by this wake. CERN's leading candidate [6] consists of two parallel accelerators where the driver consists of metallic rf structures which enables rf extraction and guiding to the acceleration component. At relatively low energies the concept was demonstrated in 1988 [7] where a test bunch was injected in a wakefield and according to its location relative to the drive bunch, it was shown that the test bunch can be accelerated or decelerated.

We suggest a new paradigm of TBA which alleviates the necessity of a high-charge drive bunch. Instead, in our configuration a low-charge bunch triggers a weak intensity Cherenkov wake in a solid-state active medium. The wakefield is amplified at the expense of the energy stored in the active medium. This causes the wake component to grow exponentially behind the trigger bunch, hence the main source of energy is effectively the medium rather than the bunch alone; for this reason we prefer the notion of trigger bunch in this paradigm rather than drive-bunch in the regular TBA. As the wake depletes the energy from the medium, the process reaches saturation, typically a few centimeters behind the trigger bunch. At saturation the acceleration field is maximal, thus a trailing bunch should be injected at the proper delay allowing it to experience this maximal accelerating gradient.

Previous studies presented the underlining theory for the active medium TBA, the focus was on a gaseous active medium [8-10]. The latter has one major drawback: for storing enough energy in order to be competitive with other acceleration schemes, high gas pressure is required and this leads to significant small-angle electron scattering which is detrimental to the transverse emittance of the accelerated 


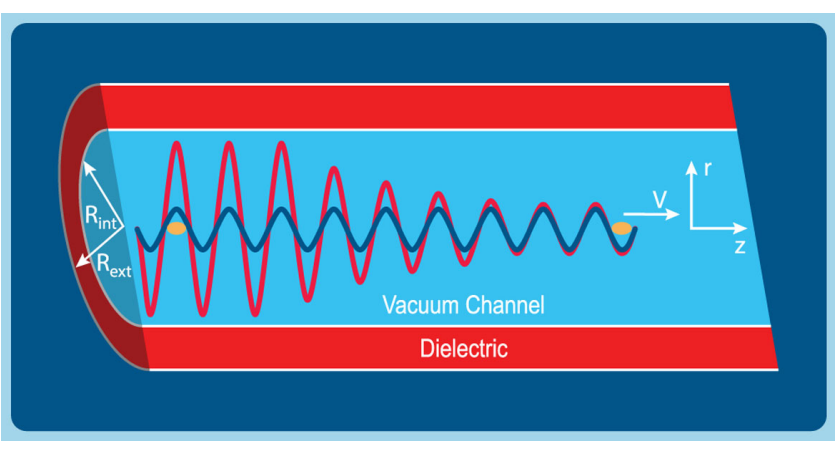

FIG. 1. Schematics of the suggested configuration. A trigger electron bunch (yellow) with velocity $\mathrm{V}$ is injected into a vacuum channel with radius $R_{\text {int }}$ of a dielectric medium cylinder with external radius $R_{\text {ext }}$ (the figure shows a cutting place of the geometry). The bunch generates a Cherenkov wake which can accelerate a trailing bunch. In the case of a passive dielectric medium, the wake has a constant amplitude (dark blue). In the case of an active dielectric medium, the wake is amplified by the medium (dark red) and exponentially grows behind the trigger electron bunch. The drawing is not up to scale.

beam. An additional drawback is the ionization of atoms in the gas; they may also enhance the small-angle scattering. In this study we propose a solid-state active medium where the electrons travel in vacuum, inside an inner channel, eliminating any scattering process. Furthermore, a solidstate medium stores substantial more optical energy in a given volume. An additional advantage is the efficiency of the scheme due the highly efficient diode-pumping of a solid-state active medium, since ideally, all the stored energy is extracted in the amplification process [11] and transferred to the wake. As will be discussed, using an Nd-doped active medium, that is only millimeters thick, can yield an accelerating electric field at the range of $\sim 1 \mathrm{GV} / \mathrm{m}$.

In this study we develop a theoretical model that provides the scaling laws describing the initial excited field, growing wake and saturation in a two-beam solidstate active medium acceleration paradigm. The configuration in focus consists of a hollow cylindrical rod of an external radius $R_{\text {ext }}$ and internal radius $R_{\text {int }}$ facilitating the transport of a relativistic electron ensemble, as shown in Fig. 1. The illustration in the latter shows the wake generated behind the trigger bunch, where for a passive dielectric has a constant amplitude (dark blue) and for an active dielectric its amplitude exponentially grows (dark red). In Sec. II we formulate a linear model for the wake generation and amplification in this configuration (we moved all the noncrucial mathematical steps to two Appendixes). As a point of reference, the latter also includes a corresponding model for a passive medium. Next, in Sec. III we analyze the initiation of the wakefield, i.e., the trigger field measured at the tail of the trigger beam and in Sec. IV we examine the growth of this wakefield. In Sec. V we account for saturation of the interaction due to the limited energy stored in the medium. The parametric dependence of the gradient at saturation is described extensively. In Sec. VI we conclude our study with a practical set of parameters for future proof-of-concept experiments. Several optional configurations are discussed.

\section{LINEAR REGIME}

Let us start with the description of the model we employ for the quasianalytic analysis of the electromagnetic wake in the presence of a solid-state dielectric active medium, for the configuration of a hollow rod depicted in Fig. 1.

Outside the hollow rod there is vacuum, $\varepsilon_{r}=1$, whereas for the dielectric we assume a linear material

$$
\varepsilon(\omega)=\varepsilon_{r}+\frac{\omega_{\mathrm{pl}}^{2}}{-\omega^{2}+j \omega \Delta \omega+\omega_{\text {res }}^{2}} \equiv \varepsilon_{r}+\delta \varepsilon(\omega)
$$

where $\omega_{\text {res }}$ is the medium's resonance frequency and $\Delta \omega$ is the bandwidth. The effective plasma frequency $\omega_{\mathrm{pl}}$ is determined from the condition that for a plane wave, the gain at resonance $\omega \simeq \omega_{\text {res }}$, has the form $\exp (\alpha z)$, or explicitly $\omega_{\mathrm{pl}}=j \sqrt{2|\alpha| c \Delta \omega \sqrt{\varepsilon_{\mathrm{r}}}}$. As a figure of merit, these three parameters for Nd:YAG are: $\lambda_{\text {res }}=1.064 \mu \mathrm{m}=$ $2 \pi c / \omega_{\text {res }}, \quad \Delta \omega \sim 10^{12} \mathrm{~s}^{-1} \quad$ and $\quad|\alpha|=2 \times 10^{3} \mathrm{~m}^{-1}-$ see $\operatorname{Ref}[12]$.

In this system an azimuthally symmetric charged-loop of radius $r_{\mathrm{so}}$ moves at a velocity $c \beta$, and at $t=0$ it is located at $z_{\mathrm{so}}$. The corresponding current density is

$$
J_{z}(r, z, t)=-\frac{q_{\mathrm{el}} c \beta}{2 \pi r} \delta\left(r-r_{\mathrm{so}}\right) \delta\left(z-z_{\mathrm{so}}-c \beta t\right) .
$$

where $q_{\mathrm{el}}$ is a single electron charge and the two delta functions involved indicate that this is actually an infinitesimally thin loop. By virtue of linearity of Maxwell equations, a realistic azimuthally symmetric distribution is determined by a superposition of such sources. Given this configuration, our next step is to determine the Green's function of the system.

\section{A. Green's function}

We consider the wakefield excited by a single charged loop traveling through the vacuum channel $r_{\text {so }}<R_{\text {int }}$. The longitudinal current density induces a longitudinal magnetic vector potential which in turn, has two components: the nonhomogeneous solution, can be considered as the primary one (superscript pri) and it is driven by the current density ignoring the hollow rod. The homogeneous solution is considered to be the secondary contribution (superscript sec) and it accounts for the hollow rod. The superposition of the two components, satisfies the boundary conditions at $r=R_{\text {int }}$ and $r=R_{\text {ext }}$. Explicitly, in the absence of the hollow rod, the prescribed current density excites a primary magnetic vector potential 


$$
\begin{aligned}
A_{z}^{(\mathrm{pri})}(r, z, t)= & -\frac{q_{\mathrm{el}} \mu_{0}}{(2 \pi)^{2}} \int_{-\infty}^{\infty} d \omega \exp (j \omega \tau) \\
& \times \begin{cases}K_{0}(\Gamma r) I_{0}\left(\Gamma r_{\mathrm{so}}\right) & r>r_{\mathrm{so}} \\
K_{0}\left(\Gamma r_{\mathrm{so}}\right) I_{0}(\Gamma r) & r<r_{\mathrm{so}}\end{cases}
\end{aligned}
$$

where $c^{2} \Gamma^{2} \equiv \omega^{2} / \beta^{2} \gamma^{2}, \gamma^{-2} \equiv 1-\beta^{2}$ is the Lorentz factor, $\tau \equiv t-\left(z-z_{\mathrm{so}}\right) / c \beta$ and $I_{0}, K_{0}$ are the zero-order modified Bessel functions of the first and second kind respectively.

Accounting for the presence of a hollow rod, a secondary magnetic vector potential is induced

$$
\begin{aligned}
A_{z}^{(\mathrm{sec})}= & -\frac{q_{\mathrm{el}} \mu_{0}}{(2 \pi)^{2}} \int_{-\infty}^{\infty} d \omega \exp (j \omega \tau) \\
& \times \begin{cases}A I_{0}(\Gamma r) & 0 \leq r<\mathrm{R}_{\mathrm{int}} \\
B J_{0}(\Lambda r)+C Y_{0}(\Lambda r) & R_{\mathrm{int}}<r<R_{\mathrm{ext}} \\
D K_{0}(\Gamma r) & R_{\mathrm{ext}} \leq r<\infty\end{cases}
\end{aligned}
$$

where $c^{2} \Lambda^{2} \equiv \omega^{2} \bar{\varepsilon}, \bar{\varepsilon} \equiv \varepsilon-\beta^{-2}, J_{0}, Y_{0}$ are the zero-order Bessel functions and A,B,C,D are yet unknown amplitudes which depend on the charge, frequency, dielectric coefficient, and the geometry.

The continuity of the tangential electric and magnetic fields $E_{z}, H_{\phi}$ imposes four conditions which dictate the four unknown amplitudes. Keeping in mind the typical dimensions for the active-medium rod i.e., $R_{\text {ext }} \sim 1-4 \mathrm{~mm}, R_{\text {int }} \sim$ $10-50 \mu \mathrm{m}$ we conclude that $\Lambda R_{\text {int }} \gg 1, \Lambda R_{\text {ext }} \gg 1$ and we may replace the zero and first order of the first and second kind Bessel functions $J_{0}, J_{1}, Y_{0}, Y_{1}$ for large arguments, with their asymptotic expression; we discuss subsequently the implication of this assumption on our model. Consequently, the secondary (longitudinal) electric field in the range of the vacuum channel reads

$$
\begin{aligned}
\frac{E_{z}^{(\mathrm{sec})}(r, \tau)}{q_{\mathrm{el}} \mu_{0} / 2 \pi(\gamma \beta)^{2}}= & \frac{1}{2 \pi} \int_{-\infty}^{\infty} d \omega(j \omega) \exp (j \omega \tau) \\
& \times F_{\perp}\left(\omega^{2}\right) \frac{\sqrt{a_{11}^{2}+a_{12}^{2}}}{\sqrt{a_{21}^{2}+a_{22}^{2}}} \\
& \times \frac{\sin \left[\theta+\arctan \left(a_{12} / a_{11}\right)\right]}{\sin \left[\theta+\arctan \left(a_{22} / a_{21}\right)\right]} \\
\equiv & \frac{1}{2 \pi} \int_{-\infty}^{\infty} d \omega(j \omega) \exp (j \omega \tau) \frac{\mathcal{N}\left(\omega^{2}, \varepsilon\right)}{\mathcal{D}\left(\omega^{2}, \varepsilon\right)}
\end{aligned}
$$

where $F_{\perp}\left(\omega^{2}\right)=K_{0}\left(\Gamma R_{\text {int }}\right) I_{0}\left(\Gamma r_{\text {so }}\right) I_{0}(\Gamma r) / I_{0}\left(\Gamma R_{\text {int }}\right)$ is the transverse form factor, $\theta\left(\omega^{2}, \varepsilon\right) \equiv \Lambda\left(R_{\text {ext }}-R_{\text {int }}\right)$ and $a_{11}$, $a_{12}, a_{21}, a_{22}$ are defined in App. A.

For a time-domain and analytic evaluation of the integral in Eq. (5) we revert to Cauchy's residue theorem, which facilitates evaluation of the integral based on the poles of the integrand; these are assessed from the zeros of the denominator
$\mathcal{D}\left(\omega^{2}, \varepsilon\right) \equiv \sin \left\{\theta\left(\omega^{2}, \varepsilon\right)+\arctan \left[\frac{a_{22}\left(\omega^{2}, \varepsilon\right)}{a_{21}\left(\omega^{2}, \varepsilon\right)}\right]\right\}=0$

clearly satisfied when the argument of the trigonometric function equals $0, \pm \pi, \pm 2 \pi, \ldots$. We denote the zeros of this equation by $\omega_{n}$ with $n=0, \pm 1, \pm 2, \ldots \ldots$.

At this point we take advantage of the fact that $\varepsilon_{r} \gg|\delta \varepsilon|$ in order to determine the solution of the above equation in two steps: in the first we establish the zero-order resonances $\left(\omega_{0, n}^{2}\right)$ for the passive case $\delta \varepsilon=0$; subscript 0 differentiate between the general case solution $\omega_{n}$ and the zero-order solution, $\omega_{0, n}$. This first step is straightforward since

$$
\mathcal{D}_{0}\left(\omega_{0, n}^{2}\right) \equiv \mathcal{D}\left(\omega_{0, n}^{2}, \varepsilon_{r}\right)=0 .
$$

In the second step $|\delta \varepsilon| \neq 0$ and we establish the frequency deviation due to this correction. For this purpose we expand the denominator in Taylor series in both variables $\left(\omega^{2}, \delta \varepsilon\right)$ up to first order in each

$$
\begin{aligned}
\mathcal{D}\left(\omega^{2}, \varepsilon\right) \simeq & \underbrace{\mathcal{D}\left(\omega_{0, n}^{2}, \varepsilon_{r}\right)}_{\mathcal{D}_{0}\left(\omega_{0, n}^{2}\right)=0}+\left[\frac{\partial \mathcal{D}}{\partial \omega^{2}}\right]_{\omega^{2}=\omega_{0, n}^{2}}\left(\omega^{2}-\omega_{0, n}^{2}\right) \\
& +\left[\frac{\partial \mathcal{D}}{\partial \varepsilon}\right]_{\substack{\omega_{r}^{2}=\omega_{0, n}^{2} \\
\varepsilon=\varepsilon_{r}}} \delta \varepsilon .
\end{aligned}
$$

With these expressions in mind, we may simplify the fraction in the integrand of Eq. (5) keeping in mind that the main contribution of the numerator is from the zero order frequency $\omega_{n}^{2} \simeq \omega_{0, n}^{2}$

$$
\frac{\mathcal{N}\left(\omega^{2}, \varepsilon\right)}{\mathcal{D}\left(\omega^{2}, \varepsilon\right)} \simeq \sum_{n} \frac{\mathcal{N}\left(\omega_{0, n}^{2}, \varepsilon_{r}\right) \mathcal{D}_{\omega^{2}}^{-1}}{\omega^{2}-\omega_{0, n}^{2}+\frac{\omega_{\mathrm{pl}, n}^{4}}{\omega_{\mathrm{res}}^{2}+j \omega \Delta \omega-\omega^{2}}}
$$

here we defined

$$
\omega_{\mathrm{pl}, n}^{4} \equiv \omega_{\mathrm{pl}}^{2} \frac{\mathcal{D}_{\varepsilon}}{\mathcal{D}_{\omega^{2}}} \simeq \omega_{\mathrm{pl}}^{2} \frac{\omega_{0, n}^{2}}{\bar{\varepsilon}_{r}}
$$

and $\bar{\varepsilon}_{r} \equiv \varepsilon_{r}-\beta^{-2}, \mathcal{D}_{\varepsilon}$ as well as $\mathcal{D}_{\omega^{2}}$ represent the first derivative with respect to the corresponding subscript, evaluated at $\varepsilon=\varepsilon_{r}$ and $\omega^{2}=\omega_{0, n}^{2}$.

For these simplifications we may write the integral representation of Green's function as

$$
\begin{aligned}
G_{n}(\tau) & \equiv \frac{1}{2 \pi} \int_{-\infty}^{\infty} d \omega \exp (j \omega \tau) \frac{-\omega^{2}+j \omega \Delta \omega+\omega_{\mathrm{res}}^{2}}{\mathcal{D}_{G, n}(\omega)}, \\
\mathcal{D}_{G, n}(\omega) & \equiv\left(\omega^{2}-\omega_{0, n}^{2}\right)\left(-\omega^{2}+j \omega \Delta \omega+\omega_{\mathrm{res}}^{2}\right)+\omega_{\mathrm{pl}, n}^{4}
\end{aligned}
$$

The latter is a fourth order polynomial in $j \omega$ with 4 solutions which are denoted by $s_{n, i}$ with $i=1,2,3,4$. Employing the fact that $f(t) \equiv(1 / 2 \pi) \int_{-\infty}^{\infty} d \omega \exp (j \omega t) /(j \omega-s)=$ $\exp (s t) h(t)$, where $h(t)$ is the Heaviside step function, we get an exact time-domain solution including the solution 
which grows in time in case of an active medium. It is tacitly assumed that this growth is limited in time since the total energy stored in the medium is finite-we quantify this subsequently when we elaborate on the saturation of the system. Subject to this limitation, the longitudinal electric field reads,

$E_{z}^{(\mathrm{sec})}=E_{0}^{(\mathrm{sec})} \sum_{n} \bar{a}\left(\omega_{0, n}\right) F_{\perp}\left(\omega_{0, n}^{2}\right) \sum_{i=1}^{4} b_{n, i} s_{n, i} \exp \left(s_{n, i} \tau\right) h(\tau)$

$E_{0}^{(\mathrm{sec})} \equiv \frac{q_{\mathrm{el}}}{4 \pi \varepsilon_{0}\left(R_{\mathrm{ext}}-R_{\mathrm{int}}\right)^{2}} \frac{2}{(\gamma \beta)^{2} \sqrt{\bar{\varepsilon}_{r}}}$

where $b_{n, i}$, the amplitudes dictated by the zeros $s_{n, i}$, and $\bar{a}\left(\omega_{0, n}\right)$ are given in Appendix A. Equation (12) describes the time domain longitudinal wakefield excited by a single charged loop which traverses an active medium through a vacuum channel in its core. Evidently, the wakefield consists of a combination of $n$ modes, however we are interested only in the modes which can interact with the medium and gain energy, i.e., modes which satisfy $\left|\omega_{0, n}\right| \simeq \omega_{\text {res }}$.

The wakefield trailing the electrons (for $\tau>0$ ) is exponentially dependent on the poles $s_{n, i}$, which near the resonance frequency have the form $s_{n, i} \simeq \pm j \omega_{\text {res }} \pm \delta \omega$. Assuming negligible bandwidth $\left(\Delta \omega \ll \omega_{\text {res }}\right)$, the term $\delta \omega$ is roughly $\delta \omega \simeq \sqrt{\frac{1}{2} \alpha c \Delta \omega \sqrt{\varepsilon_{r}} / \bar{\varepsilon}}$. This form implies that one of the four solutions $s_{n, i}$ is a growing mode, oscillating according to $\sim \omega_{\text {res }}$ with a gain parameter $\delta \omega$. In what follows, the focus of our analysis is in these growing modes, as they dominate the interaction. It is important to point out that the growing mode is a solution given by the linear model, not accounting for the non-linear saturation; the latter will be discussed in Sec. V.

\section{B. Electron bunch}

Once we have determined Green's function for wakefield generated by a single charged loop (delta like excitation) in an active medium, we can proceed to establish the wake of a finite-size circular trigger bunch containing $N_{\text {tg }}$ electrons. We consider uniform distributions in both radial and longitudinal directions. In addition, the two distributions are assumed to be statistically independent; its length is denoted by $c \Delta_{\operatorname{tg}}$ whereas its radius is $R_{\mathrm{tg}}$. To account for the field induced by the trigger bunch we integrate over the initial location $\left(r_{\mathrm{so}}, z_{\mathrm{so}}\right)$. Behind the trigger bunch $\tilde{\tau} \equiv t-z / c \beta>\Delta_{\mathrm{tg}} / 2$, this field reads

$$
\begin{aligned}
\left\langle E_{z}^{(\mathrm{sec})}(\tilde{\tau}, r)\right\rangle= & N_{\mathrm{tg}} E_{0}^{(\mathrm{sec})} \sum_{n} \bar{a}\left(\omega_{0, n}\right) \\
& \times \frac{K_{0}\left(\Gamma_{n} R_{\mathrm{int}}\right)}{I_{0}\left(\Gamma_{n} R_{\mathrm{int}}\right)} I_{c}\left(\Gamma_{n} R_{\mathrm{tg}}\right) I_{0}\left(\Gamma_{n} r\right) \\
& \times \sum_{i=1}^{4} b_{n, i} s_{n, i} e^{s_{n, i}} \tilde{\operatorname{\tau }} \operatorname{sinch}\left(s_{n, i} \frac{\Delta_{\mathrm{tg}}}{2}\right)
\end{aligned}
$$

here $c^{2} \Gamma_{n}^{2} \equiv \omega_{0, n}^{2} / \beta^{2} \gamma^{2}, I_{c}(x) \equiv 2 I_{1}(x) / x$ and $\operatorname{sinch}(x) \equiv$ $\sinh (x) / x$. The field given in Eq. (13) can be used as an accelerating field for a second bunch, i.e., a trailing bunch (subscript tr) injected behind the trigger bunch at a given delay. The average field experienced by such trailing bunch with uniform distribution, a radius $R_{\text {tr }}$ and length $c \Delta_{\text {tr }}$ is given by

$$
\begin{aligned}
\left\langle E_{z}^{(\mathrm{sec})}(\tilde{\tau})\right\rangle= & N_{\mathrm{tg}} E_{0}^{(\mathrm{sec})} \sum_{n} \bar{a}\left(\omega_{0, n}\right) \\
& \times \frac{K_{0}\left(\Gamma_{n} R_{\mathrm{int}}\right)}{I_{0}\left(\Gamma_{n} R_{\mathrm{int}}\right)} I_{c}\left(\Gamma R_{\mathrm{tr}}\right) I_{c}\left(\Gamma R_{\mathrm{tg}}\right) \\
& \times \sum_{i=1}^{4} b_{n, i} s_{n, i} e^{s_{n, i}} \operatorname{sinch}\left(s_{n, i} \frac{\Delta_{\mathrm{tg}}}{2}\right) \\
& \times \operatorname{sinch}\left(s_{n, i} \frac{\Delta_{\mathrm{tr}}}{2}\right)
\end{aligned}
$$

where we averaged over the field radial cross section and duration.

It is important to emphasize that the approach presented here is applicable for a large variety of configurations such as Bragg structure [13], photonic band-gap [14] or dielectric grating [15]. The numerator $\mathcal{N}$ and the denominator $\mathcal{D}$ as introduced in Eq. (5) may have a different geometry dependence but the 4th order polynomial has the same form in all configurations.

\section{Passive medium}

The validity of the approach may be readily exemplified on a passive medium $[16,17]$. Here we consider a medium with a constant dielectric coefficient $\varepsilon=\varepsilon_{r}$, rather than the frequency dependent $\varepsilon(\omega)$ of an active medium.

The starting point for the passive medium analysis is the secondary longitudinal field in Eq. (5), where the full expression for the denominator is given in Eq. (6). The Taylor expansion of the latter, previously given in Eq. (8) is now reduced to

$\mathcal{D}^{\text {pass }}\left(\omega^{2}, \varepsilon\right) \simeq \underbrace{\mathcal{D}\left(\omega_{0, n}^{2}, \varepsilon_{r}\right)}_{\mathcal{D}_{0}\left(\omega_{0, n}^{2}\right)=0}+\left[\frac{\partial \mathcal{D}}{\partial \omega^{2}}\right]_{\substack{\omega^{2}=\omega_{0, n}^{2} \\ \varepsilon=\varepsilon_{r}}}\left(\omega^{2}-\omega_{0, n}^{2}\right)$

as $\delta \varepsilon(\omega) \equiv 0$. This leads us to the passive case Green's function

$G_{n}^{\mathrm{pass}}(\tau) \equiv \frac{1}{2 \pi} \int_{-\infty}^{\infty} d \omega \frac{\exp (j \omega \tau)}{\omega^{2}-\omega_{0, n}^{2}}=-\frac{1}{\omega_{0, n}} \sin \left(\omega_{0, n} \tau\right) h(\tau)$

where clearly, there are two groups of solutions $\omega= \pm \omega_{0,|n|}$ and not four groups as in the active medium scenario. Consequently, the longitudinal electric field induced by a single charged loop reads 


$$
\begin{aligned}
E_{z, \text { pass }}^{(\mathrm{sec})}\left(r, \tau ; r_{\mathrm{so}}\right)= & E_{0}^{(\mathrm{sec})} \sum_{n} \bar{a}\left(\omega_{0, n}\right) F_{\perp}\left(\omega_{0, n}^{2}\right) \\
& \times \cos \left(\omega_{0, n} \tau\right) h(\tau)
\end{aligned}
$$

where the corresponding wake trailing a uniformly distributed circular electron bunch is given by

$$
\begin{aligned}
\left\langle E_{z, \mathrm{pass}}^{(\mathrm{sec})}(\tilde{\tau}, r)\right\rangle= & N_{\mathrm{tg}} E_{0}^{(\mathrm{sec})} \sum_{n} \bar{a}\left(\omega_{0, n}\right) \\
& \times \frac{K_{0}\left(\Gamma_{n} R_{\mathrm{int}}\right)}{I_{0}\left(\Gamma_{n} R_{\mathrm{int}}\right)} I_{c}\left(\Gamma_{n} R_{\mathrm{tg}}\right) I_{0}\left(\Gamma_{n} r\right) \\
& \times \cos \left(\omega_{0, n} \tilde{\tau}\right) \operatorname{sinc}\left(\omega_{0, n} \frac{\Delta_{t g}}{2}\right)
\end{aligned}
$$

Evidently, the modes in a passive medium are oscillating rather than growing/decaying. The contributing modes are dictated by the Cherenkov condition $\sqrt{\varepsilon_{r}} \beta>1$, where below this condition the modes $\omega_{0,|n|}$ [dictated by Eq. (6)] are pure imaginary, thus resulting in evanescent waves.

We note that the radial dependence of all modes, in the passive and active cases, is determined by $F_{\perp}\left(\omega_{0, n}^{2}\right) \propto$ $I_{0}\left(\Gamma_{n} r\right)$, indicating the wake is strongest on the boundary $R_{\text {int }}$ and decaying toward the center of the vacuum channel as the Cherenkov wake is excited inside the dielectric medium.

In order to further test our analytic approach and illustrate the excitation of the Cherenkov wake in the hollow rod configuration, we perform simulations in CST Particle Studio (www.cst.com), employing the wakefield solver, for the passive medium case. It should be emphasized that CST currently does not support active medium simulations. The parameters for the subsequent analysis were chosen according to several guidelines. First, the analytic model in Sec. II assumes $\Lambda R_{\text {int }} \gg 1, \Lambda R_{\text {ext }} \gg 1$, hence the geometrical parameters considered must comply with this condition. Second, in order to simulate an infinite vacuum surrounding, PML (perfect matching layer) boundaries are required. However, due to limitations associated with PML [18], we surround the dielectric rod with a PEC (perfect electric conductor). For the latter case, the resonance frequencies are determined by

$\tilde{\mathcal{D}}\left(\omega^{2}, \varepsilon\right) \equiv \sin \left\{\theta\left(\omega^{2}, \varepsilon\right)-\arctan \left[\frac{1}{\tilde{a}\left(\omega^{2}, \varepsilon\right)}\right]\right\}=0$

where $\tilde{a}\left(\omega^{2}, \varepsilon\right)=\zeta I_{1}\left(\Gamma R_{\text {int }}\right) / I_{0}\left(\Gamma R_{\text {int }}\right)$. We find that the resonance frequencies dictated by Eq. (19) as compared with the resonance frequencies dictated by Eq. (6), for the case of vacuum surrounding, are different by less than 1 percent at the optical range. Additionally, the radiation transmitted through the vacuum-dielectric interface exponentially decays, thus effectively no power propagates radially. Therefore, the PEC surrounded configuration may serve as a good measure for our analytic model.

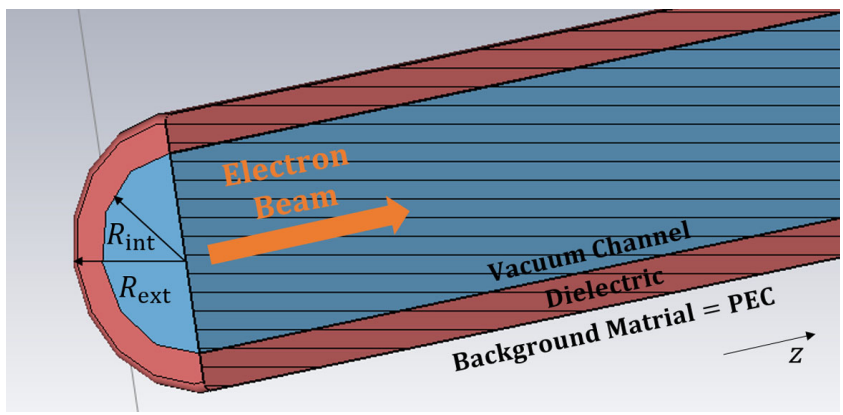

FIG. 2. Simulated configuration.

The simulated structure is depicted in Fig. 2, where we employed a hexahedral mesh with 30 lines per wavelength, which is sufficient for convergence [19].

According to the analytical model, when considering Gaussian longitudinal spread of the trigger bunch, corresponding to the CST simulation source, the longitudinal field trailing the trigger bunch on-axis is given by

$$
\begin{aligned}
& E_{z, \text { passive }}^{\text {Gauss(sec) }}=N_{\mathrm{tg}} E_{0}^{(\mathrm{sec})} \sum_{n}\left[F_{\perp}\left(\omega_{0, n}^{2}\right)\right]_{\substack{r=0 \\
r_{\mathrm{s} 0}=0}} \bar{a}\left(\omega_{0, n}\right) \\
& \times \exp \left(-\frac{\sigma_{\mathrm{tg}}^{2} \omega_{0, n}^{2}}{2}\right) \cos \left(\omega_{0, n} \tilde{\tau}\right)
\end{aligned}
$$

where $\tau_{\mathrm{so}} \equiv z_{\mathrm{so}} / c \beta$ and $\sigma_{\mathrm{tg}}$ is the bunch standard deviation.

For typical rod dimensions of $R_{\text {ext }}$ in the $\mathrm{mm}$ range, e.g., $R_{\text {ext }}=1 \mathrm{~mm}$, and $R_{\text {int }}$ in the sub-mm range, e.g $R_{\text {int }}=0.65 \mathrm{~mm}$, with $\sigma_{\mathrm{tg}} \sim 0.3 \mathrm{~mm}$, the resonance frequencies at the optical range $\left(\lambda_{n}=1 \mu \mathrm{m}\right)$ would occur at $n>1001$ [n denotes the resonance index in Eq. (6)]. In order to avoid significant numerical noise while simulating numerous resonances, we take advantage of the linearity of Maxwell equations and scale down the dimensions, choosing geometrical parameters of $R_{\mathrm{ext}}=11 \mu \mathrm{m}$ and $R_{\text {int }}=8 \mu \mathrm{m}$ where the standard deviation of the beam is $\sigma_{\mathrm{tg}}=1.43 \mu \mathrm{m}$. In this configuration the geometrical resonance corresponds to Nd:YAG internal resonance ( $f \simeq 0.28 \mathrm{PHz}$ ) at $n=10$. For testing the analytic model we will focus on the first three geometrical resonance frequencies; the simulated dielectric rod is characterized by $\varepsilon_{r}=3.312$.

Figure 3 shows the spectrum of $E_{z}$ (blue) measured at the CST field probe located at $z=100 \mu \mathrm{m}$ on axis. The vertical lines (red) indicate the three discrete resonance frequencies predicted by our analytic model for the simulated frequency range. The percentage of error for each resonance, as compared with the peaks in the CST simulation spectrum, is indicated in the figure. Evidently, the CST highest frequencies are similar to the predicted ones; the error is $7.10 \%$ for the lowest frequency, and decreases to less than 1 percent for the second and third 


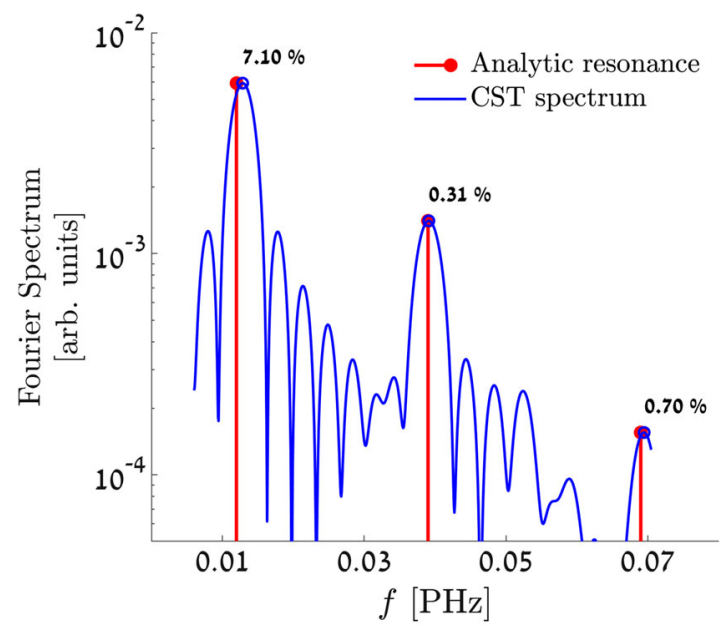

FIG. 3. Spectrum of Fourier transform of the longitudinal field $E_{z}$ (blue) measured at a probe located at $z=100 \mu \mathrm{m}$ on axis $(r=0)$. The vertical lines (red) mark the discrete resonance frequencies predicted by the analytic model at the simulated range. For a figure of merit, the internal resonance of Nd:YAG would occur at $f \simeq 0.28 \mathrm{PHz}$ (10th resonance). The percentage of error between each analytic resonance and the CST spectrum local maximum peaks are indicated in the figure (black).

resonance. This is expected, as only the latter highly agree with the assumption $\Lambda R_{\text {int }} \gg 1, \Lambda R_{\text {ext }} \gg 1$.

Next, Fig. 4 shows the normalized longitudinal electric field at the probe (blue), for a time period wherein the bunch passes thorough the probe location and propagates beyond it. The field given by the analytic model (red) is calculated according to Eq. (20) employing the three resonance frequencies determined by the CST simulation. We compare the two curves at the time period after

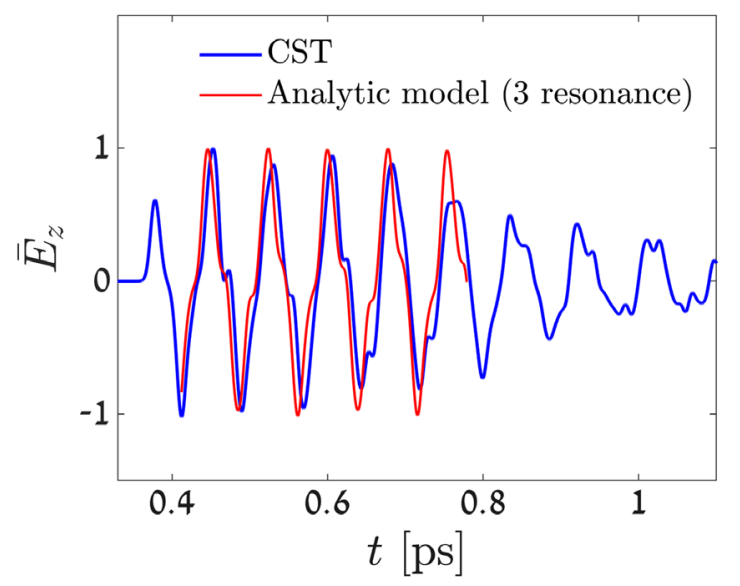

FIG. 4. Normalized longitudinal electric field $\bar{E}_{z}$ (blue) measured at a probe located at $z=100 \mu \mathrm{m}$ on axis $(r=0)$. The longitudinal field given by the analytic model (red) is calculated employing the three resonance frequencies extracted from the CST simulation according to Fig. 3.

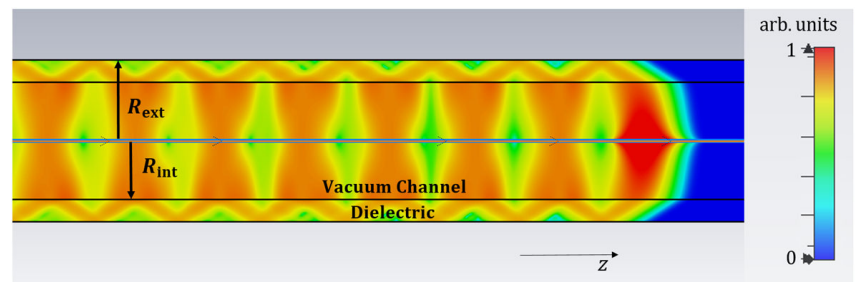

FIG. 5. The absolute value of the normalized electric field $|\bar{E}|$ at $t=1 \mathrm{ps}$, taken as a snapshot from CST.

the bunch has left the probe. As shown, the fields closely overlap, with a similar peak amplitude. Evidently, the simulated longitudinal electric field has a slowly decaying profile, this is due to transition radiation generated at the entrance [20], which is not accounted for in the analytic model. We note that the resonance frequencies which appear in Fig. 3 were calculated for the time period prior to the interference with transition radiation.

For a proper visualization of the propagating wake, Fig. 5 shows a 2D snapshot of the absolute value of the normalized field $|\bar{E}|$ at time $t=1 \mathrm{ps}$, where the beam is at $\sim 300 \mu \mathrm{m}$.

\section{TRIGGER FIELD}

For the Cherenkov wake, the field is zero in front of the trigger bunch and it reaches a certain value at its end; we refer to this initial value as the trigger field $\left|E_{z}^{(0)}\right|$ calculated according to Eq. (13), measured at the tail of the trigger bunch where $\tilde{\tau}=\tau_{0} \equiv \Delta_{t g} / 2$ and averaged over the radial cross section of the vacuum channel. Equation (13) clearly indicates a direct proportion to $N_{\text {tg }}$, while the dependency on geometrical parameters and electron energy is not straightforward. In what follows we explore the dependence of the trigger field on the important parameters for $\tau_{0}=4 \mathrm{ps}$, where $N_{\mathrm{tg}}=10^{4}$ and $\gamma=10^{4}$. The mode considered here is the closest to resonance for $\mathrm{Nd}$ : YAG, $\omega_{0,|n|} \simeq \omega_{\text {res }}$.

Figure 6(a) shows the trigger field as a function of $R_{\text {int }}$ for fixed $R_{\text {ext }}=4 \mathrm{~mm}$. We find that increasing $R_{\text {int }}$ in the range of a few microns to hundreds of microns leads to a significant decrease of the trigger field, where the curve indicates $\left|E_{z}^{(0)}\right| \propto R_{\text {int }}^{-3}$. Fig. 6b shows the dependence of the field on $R_{\text {ext }}$, for $R_{\text {int }}=10 \mu \mathrm{m}$. The curve scales like $\left|E_{z}^{(0)}\right| \propto R_{\text {ext }}^{-1}$. Figure 6(c) shows the trigger field as a function of $\Delta_{\mathrm{tg}}$ for $R_{\text {int }}=10 \mu \mathrm{m}$ and $R_{\text {ext }}=4 \mathrm{~mm}$. The trigger field remains at the same scale for values of $\Delta_{\mathrm{tg}}<10 \mathrm{ps}$, while it slowly increases for higher values. The curve scales as $\left|E_{z}^{(0)}\right| \propto \sqrt{1+\left(\Delta_{\mathrm{tg}} / \Delta_{\mathrm{tc}}\right)^{6}}$ where $\Delta_{\mathrm{tc}}=16$ ps.

Moreover, we find that the trigger field is virtually $\gamma$ independent as the change in trigger field magnitude 


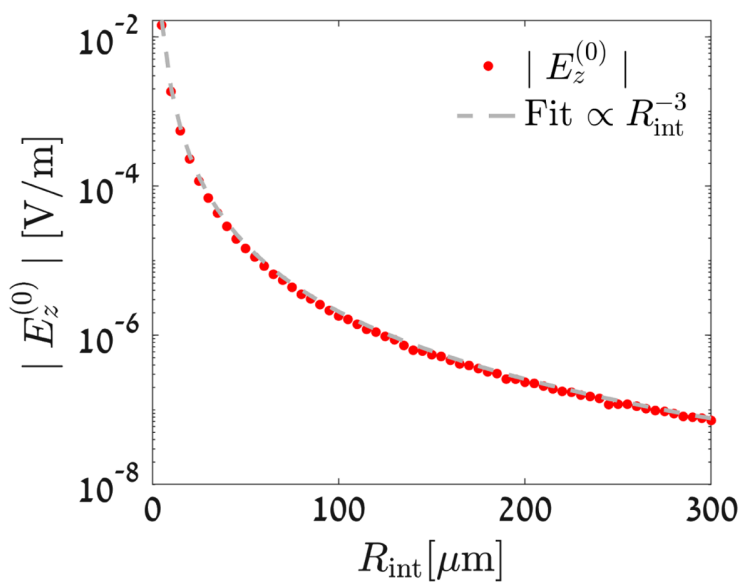

(a)

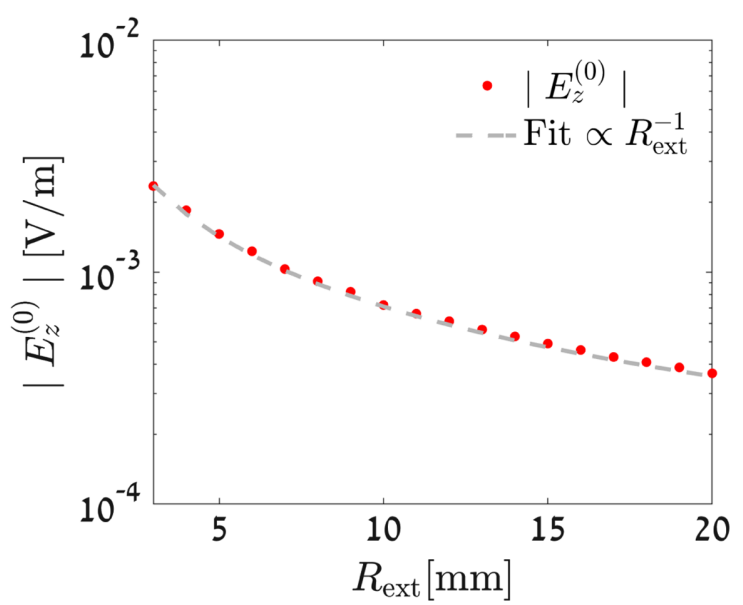

(b)

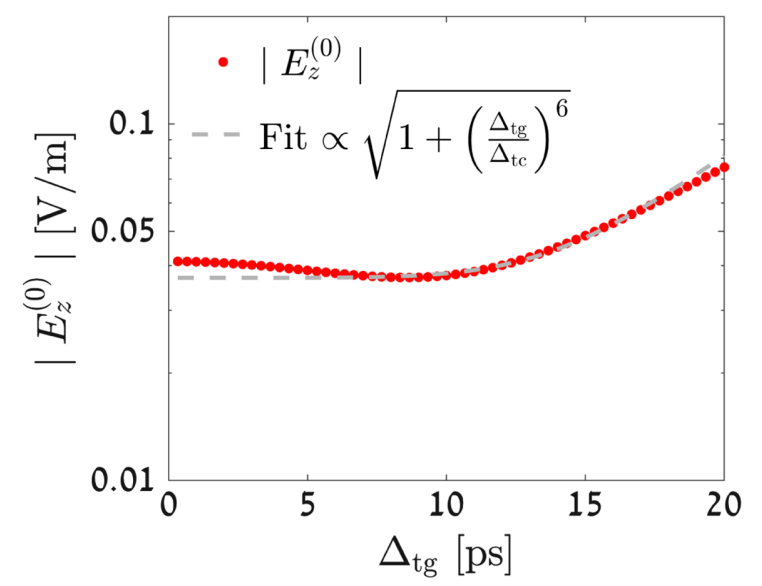

(c)

FIG. 6. Trigger field vs the configuration's internal radius $R_{\text {int }}$ (a), the external radius $R_{\text {ext }}$ (b) and the trigger bunch duration (c). We consider $N_{\mathrm{tg}}=10^{4}$ and $\gamma=10^{4}$.

induced by $\gamma$ in the range $\gamma \simeq 10^{2}-10^{4}$ does not exceed 2 percents. Therefore, we can summarize that the trigger field is given by

$$
\begin{aligned}
\left|E_{z}^{(0)}\right| \simeq & 10^{-3}[\mathrm{~V} / \mathrm{m}]\left(\frac{10[\mu \mathrm{m}]}{R_{\mathrm{int}}}\right)^{3}\left(\frac{4[\mathrm{~mm}]}{R_{\mathrm{ext}}}\right) \\
& \times \sqrt{1+\left(\frac{\Delta_{\mathrm{tg}}}{16[\mathrm{ps}]}\right)^{6}\left(\frac{N_{\mathrm{tg}}}{10^{4}}\right)}
\end{aligned}
$$

for $\gamma=10^{4}$. We emphasize that Eq. (21) considers the trigger field induced by the configuration resonance mode with frequency closest to the resonance of Nd:YAG.

\section{WAKEFIELD GROWTH}

In our analysis, the active medium is determined by the resonant frequency $\omega_{\text {res }}$, the bandwidth $(\Delta \omega)$ and the gain factor $\alpha=\Delta N \sigma_{\mathrm{SE}}$, where $\Delta N$ is the population inversion density and $\sigma_{\mathrm{SE}}$ is the stimulated emission cross section. It is important to emphasize that while these parameters were developed in the literature subject to the tacit assumption that the medium interacts with a homogeneous plane wave, we assume that this analysis is valid for our wake analysis. This can be justified by the fact that at the atomic level, the individual atom (dipole) is much smaller than the characteristic wavelength, therefore, it makes no difference if the interaction occurs with a plane wave or an evanescent wave.

Moreover, at the macroscopic level the interaction of the medium and the electrons (their wake) is expected to be dependent on the medium's geometry $\left(R_{\mathrm{int}}, R_{\mathrm{ext}}\right)$ and on the kinetic energy of the electrons. The latter has two important regimes: if the velocity of the electrons is below Cherenkov threshold, no Cherenkov radiation is emitted and therefore (ignoring the spontaneous radiation and diffraction radiation) there is no wake to amplify. At the other extreme, if the trigger bunch is ultra relativistic, $\beta \simeq 1$, and the interaction is expected to be $\gamma$-independent. It is our goal in this section to examine the wakefield growth in the region behind the trigger electron bunch $\left(\tilde{\tau} \gg \Delta_{t g} / 2\right)$. We focus the discussion to Nd:YAG as the medium due to its extensive usage - all its relevant characteristic parameters appear in Table I, however the following analysis can be generalized to any solid-state active medium.

\section{A. Growing modes}

For a complete analysis, one must account for the combination of modes $\omega_{0, n}$ interacting with the medium. For that aim we assume a uniformly distributed circular trigger bunch with a total of $N_{\mathrm{tg}}=10^{4}$ electrons. Furthermore, $R_{\mathrm{tg}} \simeq R_{\text {int }}$ and two sets of configuration characteristics are considered: (1) parameters available at small scale facilities such as the Accelerator test facility (ATF) Brookhaven National Laboratory (BNL), which we refer to as the BNL set and (2) parameters for a large scale facility such as the future design for the International Linear Collider (ILC), which we refer to as the ILC set; all parameters are listed in Table II. The wakefield magnitude 
TABLE I. Properties of Nd:YAG.

\begin{tabular}{|c|c|c|}
\hline Parameter & Symbol & Value \\
\hline Resonance wavelength $[\mu \mathrm{m}]$ & $\lambda_{\text {res }}$ & 1.064 \\
\hline Resonance frequency [rad/s] & $\omega_{\text {res }}$ & $1.77 \times 10^{15}$ \\
\hline Bandwidth [rad/s] & $\Delta \omega$ & $\sim 10^{12}$ \\
\hline $\mathrm{Nd}\left[\right.$ atoms $\left./ \mathrm{cm}^{3}\right]$ & $N_{d}$ & $1.38 \times 10^{20}$ \\
\hline Population inversion $^{\mathrm{a}}\left[\mathrm{cm}^{-3}\right]$ & $\Delta N$ & $\sim 0.7 \times 10^{20}$ \\
\hline Stimulated emission cross section $\left[\mathrm{cm}^{2}\right]$ & $\sigma_{\mathrm{SE}}$ & $2.8 \times 10^{-19}$ \\
\hline Spontaneous fluorescence lifetime $[\mu \mathrm{s}]$ & $\tau_{s p}$ & 230 \\
\hline Small signal gain $\left[\mathrm{m}^{-1}\right]$ & $\alpha$ & $\sim 2 \times 10^{3}$ \\
\hline Plasma frequency $[\mathrm{rad} / \mathrm{s}]$ & $\omega_{\mathrm{pl}}$ & $\sim 10^{12} j$ \\
\hline Passive Dielectric coefficient & $\varepsilon_{r}$ & 3.312 \\
\hline
\end{tabular}

${ }^{\mathrm{a}}$ Refers to the transient regime value, as explained in Sec. IV B.

TABLE II. Simulation parameters; active medium considered is Nd:YAG.

\begin{tabular}{lcc}
\hline \hline & BNL & ILC \\
\hline Bunch & & \\
Radius $R_{\mathrm{b}}[\mu \mathrm{m}]$ & $50-500$ & $0.005-0.729$ \\
Length $\Delta_{z}[\mathrm{~mm}]$ & 2.3 & $0.15-0.3$ \\
Energy $[\mathrm{eV}]$ & $5 \times 10^{7}$ & $5 \times 10^{11}$ \\
$\#$ of electrons & $10^{9}$ & $2 \times 10^{10}$ \\
Dielectric rod & 1 & 1 \\
$R_{\text {ext }}[\mathrm{mm}]$ & 50 & 8 \\
$R_{\text {int }}[\mu \mathrm{m}]$ & & \\
\hline \hline
\end{tabular}

${ }^{\mathrm{a}}$ The listed numbers refer to the achievable number in the relevant facility. However, for simulations we employ $10^{4}$ electrons for the trigger bunch.

is determined according to Eq. (13) where we average the field over the radial cross-section and calculate only for the growing modes $i_{0}$.

For each set of parameters the growing resonance mode where $n=n_{\text {res }}$ is the mode with frequency closest to the resonance of the medium, i.e., $\omega_{0, n} \simeq \omega_{\text {res }}$. Figure 7 shows the contribution of several growing modes around the latter, which are indexed by $\bar{n}=n_{\text {res }}+\delta n$. The magnitude of the longitudinal electric field is calculated at a distance $z \simeq 3 \mathrm{~cm} \simeq 10^{4} \lambda_{\text {res }}$ behind the trigger bunch, where the wake has significantly grown due to the interaction with the medium. Evidently, for both sets there is clearly a singledominant mode, where the following mode closest to resonance has a contribution lower by roughly two orders of magnitude. The remainder of modes are more than 4 orders of magnitude weaker.

It should be pointed out that the number of modes that grow is determined by the bandwidth, the geometry and the real part of the dielectric coefficient. For this analysis we chose $R_{\text {ext }}=1 \mathrm{~mm}$ as it enables the optimal scenario of a single dominant interacting mode for the Nd:YAG parameters. Increasing $R_{\text {ext }}$ results in a corresponding increase in the number of dominant modes which impairs the

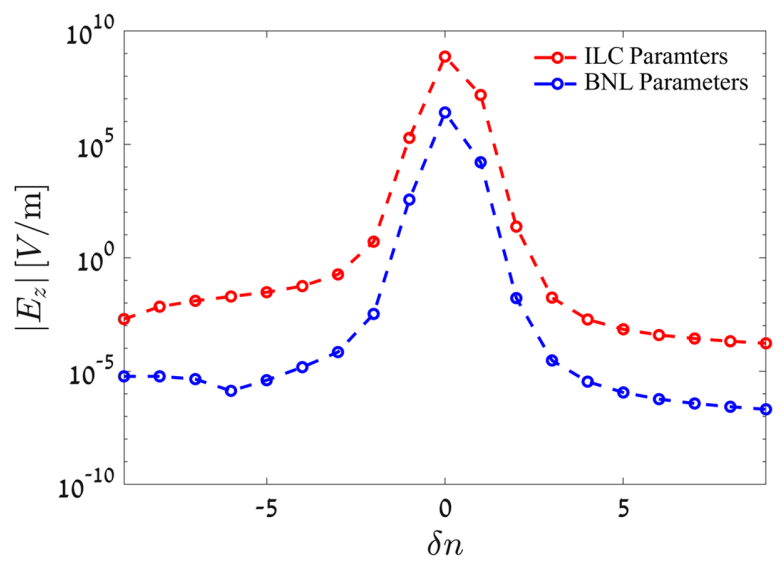

FIG. 7. Wake amplitude of near-resonance modes (centered around the resonance where $\delta n=0$ ), for the two sets of parameters: ILC set (red) and BNL set (blue). There is a single dominant mode with maximal contribution, while off-resonance modes have a contribution which is two or more orders of magnitude weaker.

acceleration process. Moreover, the combination of $R_{\text {int }}$ and $R_{\text {ext }}$ should be chosen such that one of the modes closely overlaps with the resonance frequency for maximal gain. In the following sections we consider the geometrical operation point (op): $\left(R_{\text {ext }}, R_{\text {int }}\right)=(1 \mathrm{~mm}, 8 \mu \mathrm{m})$.

\section{B. Gain parameter}

The exponential gain of the dominant mode of the wake is dictated by $\operatorname{Re}\left(s_{n_{0}, i_{0}}\right) \equiv \delta \omega$ which is dependent on the active medium parameters $\left(\omega_{0}, \Delta \omega, \omega_{\mathrm{pl}}, \varepsilon_{r}\right)$. The plasma frequency is determined by the single-pass small gain parameter $\alpha$, hence the latter also directly affects the magnitude of configuration gain.

Figure 8(a) and Fig. 8(b) show the dependency of $\delta \omega$ on $\alpha$ and $\bar{\varepsilon}_{r}$ respectively while all other medium parameters are set according to Nd:YAG, and we consider $R_{\text {ext }}=1 \mathrm{~mm}$, $R_{\text {int }}=8 \mu \mathrm{m}, \gamma=10^{4}$ and $N_{\mathrm{tg}}=10^{4}$. Evidently, for values at the range $\alpha>5 \times 10^{3}$ and $\bar{\varepsilon}_{r}>10$, the relation reads $\delta \omega \propto \sqrt{\alpha / \sqrt{\bar{\varepsilon}_{r}}}$, which is consistent with the analytic approximation, $\delta \omega \simeq \sqrt{\frac{1}{2} \alpha c \Delta \omega \sqrt{\varepsilon_{r}} / \bar{\varepsilon}_{r}}$. At smaller values, which include the parameters relevant for Nd:YAG, the dependence is stronger and the scale is given by

$$
\left(\frac{\delta \omega}{c}\right)_{\substack{\alpha<5 \times 10^{3} \\ \bar{\varepsilon}_{r}<10}} \simeq 830\left[\mathrm{~m}^{-1}\right] \frac{\alpha}{10^{3}}\left(\frac{\bar{\varepsilon}_{r}}{2.3}\right)^{-1 / 2}
$$

For the analysis we considered the regime of operation as transient [21] where the events during the wake amplification process are assumed to be fast compared with the pumping rate of the medium and the spontaneous fluorescence lifetime $\tau_{s p}$. For this case, a complete inversion of the medium is possible [22] considering state-of-the-art diode pumps. For numerical analysis we consider a moderate 


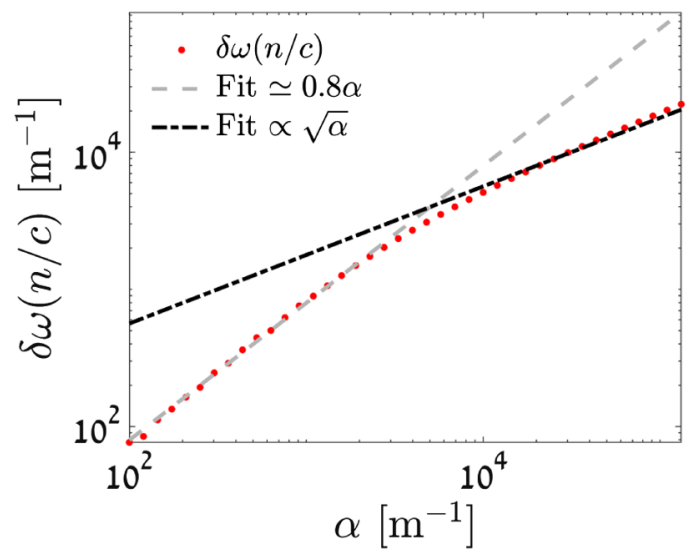

(a)

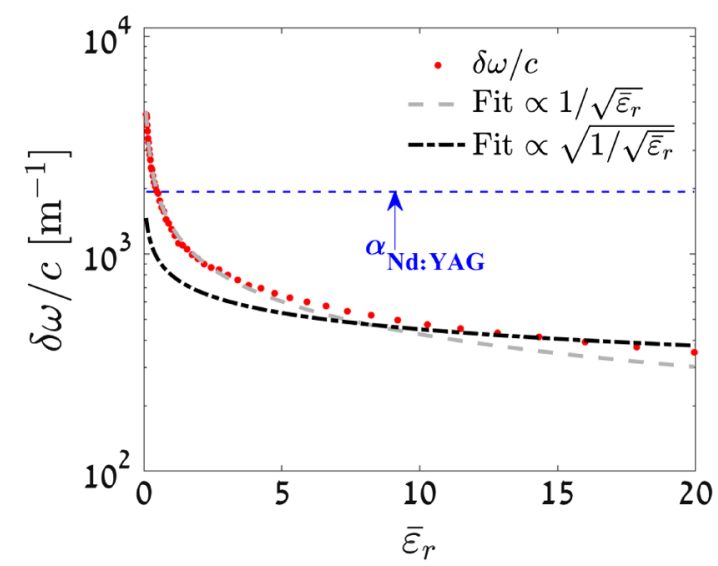

(b)

FIG. 8. Normalized exponential gain parameter $\delta \omega(n / c)$ (red) as a function of the single-pass small gain parameter $\alpha$ [8(a)] (for $\left.\bar{\varepsilon}_{r}=2.312, \mathrm{Nd}: \mathrm{YAG}\right)$, and $\delta \omega / c$ as a function of $\bar{\varepsilon}_{r}[8(\mathrm{~b})]$. Two fits are shown, one for the smaller values (gray) and another for larger values (black). The horizontal line (blue) in 8(a) represents the single-pass gain parameter $\alpha$ for Nd:YAG.

value of $\alpha \sim 2 \times 10^{3} \mathrm{~m}^{-1}$ for $\mathrm{Nd}$ YYAG, corresponding to $\sim 50 \%$ inversion [23].

We find that altering the configuration parameters $\left(R_{\text {int }}, R_{\text {ext }}, \gamma\right)$ leads to less than 3 percent change in the growth rate; it remains on the scale of $\operatorname{Re}\left(s_{n_{0}, i_{0}}\right) / c \equiv$ $\delta \omega / c \simeq 830 \mathrm{~m}^{-1}$ for the dominant modes $\omega_{0, n} \simeq \omega_{\text {res }}$ and Nd:YAG parameters.

Figure 9 illustrates the growth of $\left|E_{z}\right|$ for the dominant mode of the wake, over a distance behind the trigger bunch. We consider the two sets of parameters, BNL set and ILC set, however for both cases we took a low-charge trigger bunch of $N_{\mathrm{tg}}=10^{4}$. We simulate according to Eq. (13), averaged over the radial cross section of a trailing beam with $R_{\mathrm{tg}} \sim R_{\text {int }}$. In both configurations the exponential growth is clear and the growth rates are similar as expected. The difference in magnitudes is due to the different trigger fields, mainly dictated by $R_{\text {int }}^{-3}$ as shown in Fig. 6(a); the

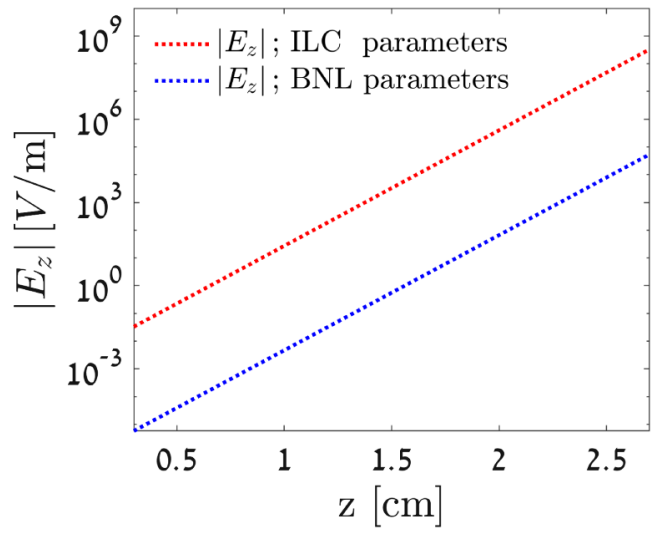

FIG. 9. Amplitude of $\left|E_{z}\right|$ behind the trigger electron bunch for ILC set (red) and BNL set (blue).

internal radius is limited by the minimal beam radius possible at each facility.

\section{GRADIENT AT SATURATION}

Taking a snapshot of the interaction process we anticipate three regimes: the first is the Cherenkov radiation build-up discussed in Sec. III, in which the amplitude of the various modes starts from zero in the front of the trigger bunch and according to the latter's length it reaches the value of the trigger field at the trigger bunch tail. In the second regime, as discussed in Sec. IV, the amplitude of the interacting mode(s) grows exponentially according to the gain factor. In the third regime, the wake field drains all the energy from the active medium and its amplitude no longer varies in space. Since in this regime the accelerating gradient reaches its maximum value, we focus in this section on the assessment of the saturation value of the field assuming that the non-linear regime does not affect the modal geometrical structure.

By virtue of the linearity of Maxwell equations, the average in time electromagnetic energy $W_{\mathrm{EM}}(z)$ per unit length in a given plane $z=$ const. is quadratic in the amplitude of the interacting mode regardless if the interaction takes place or not. Since we are interested in the longitudinal field $\left|E_{z}(z)\right|^{2}=\left(2 / R_{\mathrm{tr}}^{2}\right) \int_{0}^{R_{\mathrm{tr}}} d r r\left|E_{z}(z, r, \omega)\right|^{2}$, it is natural to define the interaction dielectric coefficient $\varepsilon_{\text {int }}$ as the ratio of the two quantities

$$
\varepsilon_{\mathrm{int}} \equiv \frac{W_{\mathrm{EM}}(z, \omega)}{\frac{1}{2} \varepsilon_{0}\left|E_{z}(z, \omega)\right|^{2} \pi R_{\mathrm{tr}}{ }^{2}}
$$

where $R_{\mathrm{tr}}$ is radius of the trailing beam, where the wake is evaluated. Three comments are in place here: (i) $\varepsilon_{\text {int }}$ resembles Pierce's parameter in traveling wave tubes and free electron lasers. (ii) for evaluation of $\varepsilon_{\text {int }}$ it is sufficient to consider the eigen-mode which corresponds to $k=\omega_{\text {res }} / c \beta$. (iii) We assume next that this quantity is valid also at the transition to saturation. 


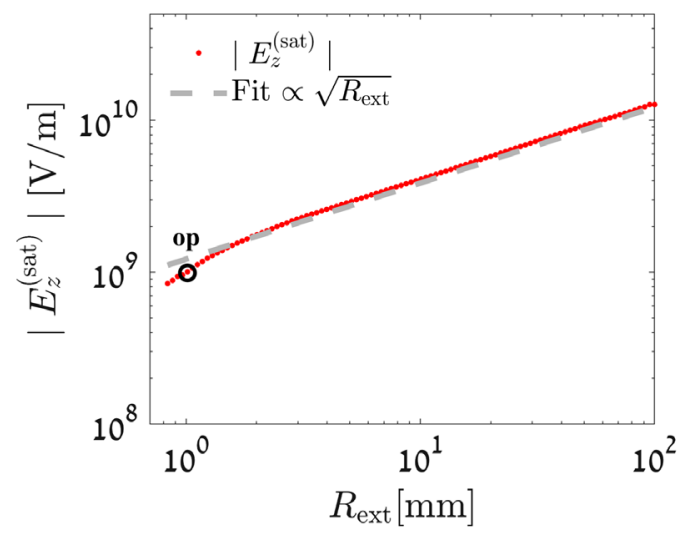

FIG. 10. Longitudinal electric field at saturation (red) vs the external radius $R_{\text {ext }}$. The fit (gray) shows a relation of $\left|E^{(\mathrm{sat})}\right| \propto \sqrt{R_{\mathrm{ext}}}$.

Once $\varepsilon_{\text {int }}$ is established, we can readily determine the gradient at saturation since the energy per unit length stored in the medium is determined by the population inversion density $\Delta N$, the energy of a single photon $\hbar \omega_{\text {res }}$ and given by $W_{\text {stored }}=\frac{1}{2} \Delta N \hbar \omega_{\text {res }} \pi\left(R_{\text {ext }}^{2}-R_{\text {int }}^{2}\right)$ [12],

$$
\left|E_{z}^{(\mathrm{sat})}\right|=\sqrt{W_{\text {stored }}\left(\frac{1}{2} \varepsilon_{\mathrm{int}} \varepsilon_{0} \pi R_{\mathrm{tr}}^{2}\right)^{-1}}
$$

where nonlinear scattering loss effects are neglected.

In the examples that follow, we assume that when saturation is approached, the interacting mode is dominant. If this is not ensured the design is not relevant to an acceleration process. Moreover, we conceive the amplitude of the field at saturation as the maximum gradient achievable $\left|E_{z}^{\mathrm{sat}}\right|=\left|E_{z}^{\max }\right|$.

In the remainder of this section we investigate the saturation gradient as a function of the major parameters $\gamma, R_{\text {int }}$ and $R_{\text {ext }}$ while the active medium is Nd:YAG. The trailing bunch is assumed to fill the entire channel $R_{\mathrm{tr}} \simeq R_{\mathrm{int}}$ thus $I_{0}\left(\Gamma_{n} r\right) \rightarrow I_{\mathrm{c}}\left(\Gamma R_{\mathrm{tr}}\right)$.

First, we examine the maximum gradient at saturation as a function of the configuration dimensions. Figure 10 shows the gradient at saturation as a function of $R_{\text {ext }}$ for $R_{\text {int }}=$ $8 \mu \mathrm{m}$ and $\gamma=10^{4}$. Since the amount of energy stored in the medium is proportional to $R_{\mathrm{ext}}^{2}$ we anticipate the gradient to increase as a function of $R_{\text {ext }}$. According to the simulation, the gradient at saturation indicates $\left|E_{z}^{(\text {sat })}\right| \propto \sqrt{R_{\text {ext }}}$. Our operation point (op) is marked on the curve.

Figure 11 shows the gradient at saturation as a function of $R_{\text {int }}$ in the range $R_{\text {int }}=1 \mu \mathrm{m}$ to $1 \mathrm{~mm}$ for $R_{\mathrm{ext}}=1 \mathrm{~mm}$ and $\gamma=10^{4}$. The plot reveals that the maximum gradient scales like $\left|E^{(\text {sat })}\right| \propto R_{\text {int }}{ }^{-3 / 2}$. The insert of Fig. 11 shows a magnification of the curve at the range of $20 \mu \mathrm{m}$ to indicate the dependency has an oscillating pattern which is induced by the Bessel functions expressions (e.g $J_{0}\left(\Lambda_{n} R_{\text {int }}\right)$ ).

Next, we analyze the gradient at saturation dependency on the trigger bunch energy $\gamma$. Figure 12 shows $\left|E_{z}^{(\text {sat })}\right|$ as a

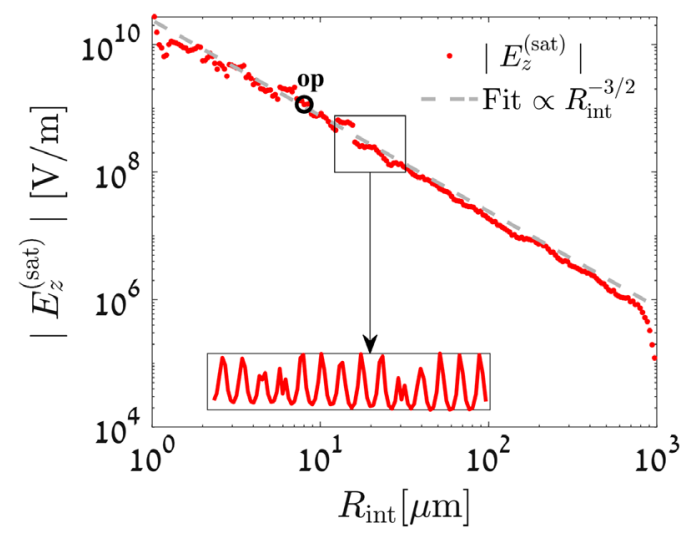

FIG. 11. Longitudinal electric field (red) at saturation vs the vacuum channel radii $R_{\text {int }}$. The fit (gray) shows the dependency is approximately $\left|E^{(\text {sat })}\right| \propto R_{\text {int }}{ }^{-3 / 2}$. The insert in the figure shows a magnification of the plot around $20 \mu \mathrm{m}$ which indicates that the jitter in the main plot is not noise, but an oscillating pattern induced by the Bessel functions in the field expressions.

function of $\gamma$ for three different values of $R_{\text {ext }}$ while keeping a fixed value of $R_{\text {int }}=8 \mu \mathrm{m}$. Evidently, the saturation gradient is $\gamma$ independent for a range of values, up to a certain critic $\gamma_{c}$ (marked in gray circles) where it gradually drops according to $\left|E_{z}^{\text {(sat) }}\right|_{\gamma>\gamma_{c}} \propto \gamma^{-2}$. The critical value is reached when a significant amount of electromagnetic energy occupies the region outside the dielectric structure, according to the spatial distribution of the mode. As can be inferred from Eq. (4), this occurs when the value of $K_{0}(\Gamma r)$, which dictates the magnitude of the field in the surrounding vacuum area, exceeds the value of $I_{0}(\Gamma r)$ which in turn, dictates the magnitude inside the vacuum channel. From the critic point on, the decrease in $\left|E_{z}^{\text {(sat) }}\right|$ is dominated by the relations between the total electromagnetic energy in the channel as compared with the energy at the outside

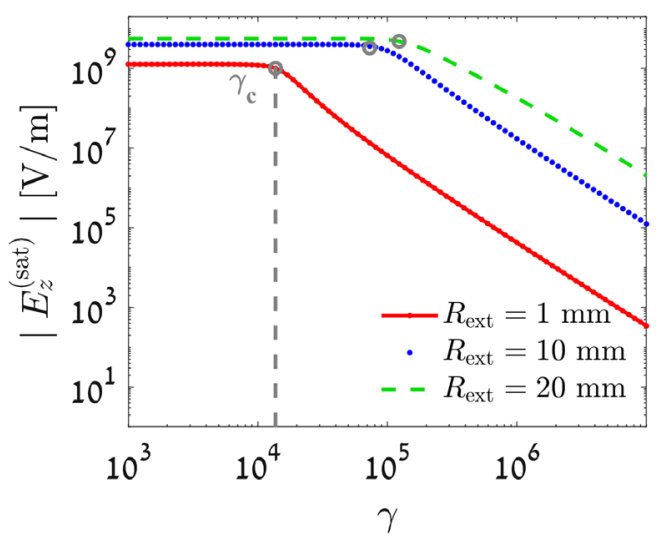

FIG. 12. Longitudinal electric field at saturation vs trigger electron bunch energy $\gamma$ for three different external rod radii. The field magnitude for all cases is constant until a critic value at the range of $\gamma_{c}>10^{4}$. 


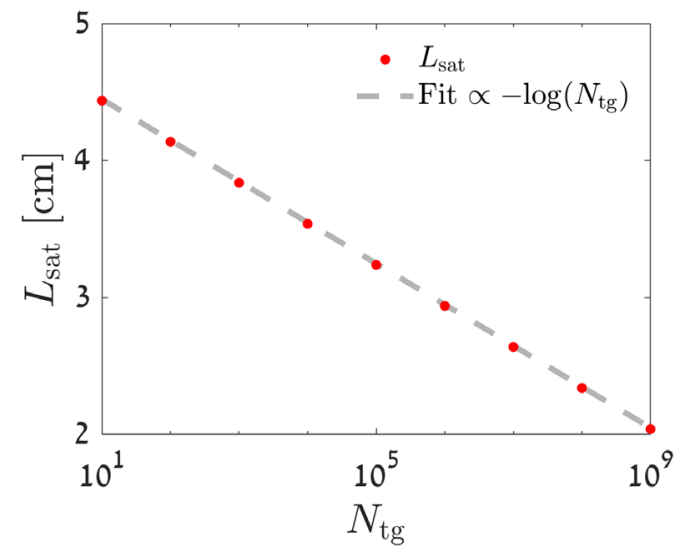

FIG. 13. Saturation length $L_{\text {sat }}$ (red) vs the number of electrons in the trigger bunch $N_{\text {tg }}$. The fit (gray) shows a relation of $L_{\mathrm{sat}} \propto-\log \left(N_{\mathrm{tg}}\right)$.

vacuum, according to $I_{1}\left(\Gamma R_{\text {int }}\right) / K_{1}\left(\Gamma R_{\text {ext }}\right) \propto \gamma^{-2}$ [see Eqs. (B2), (B3)].

Moreover, Fig. 12 reveals that different $R_{\text {ext }}$ values dictate different critical energies. We need to keep in mind that for a constant density of population inversion, the amount of stored energy which is transferred to the wake, increases with $R_{\text {ext }}$, while the energy in the surrounding vacuum (evanescent wave) increases with $\gamma ; \gamma_{c}$ represents the boundary between these two regimes. In Sec. VII we discuss a possible confining structure to reduce the spread of energy outside the configuration.

Consequently, we may conclude from the last three figures that the maximal gradient at saturation, for $\gamma<\gamma_{c}$ scales

$$
\left|E_{z}^{(\mathrm{sat}, \mathrm{max})}\right| \simeq 1.2[\mathrm{GV} / \mathrm{m}] \sqrt{\frac{R_{\mathrm{ext}}}{1[\mathrm{~mm}]}\left(\frac{8[\mu \mathrm{m}]}{R_{\mathrm{int}}}\right)^{3}}
$$

where we consider here the optional operation point for a $\mathrm{GV} / \mathrm{m}$ gradient at saturation. For this point at $\gamma<\gamma_{c}$ the acceleration gradient reaches its maximal value at $\sim 3 \mathrm{~cm}$. With this relation we can now encapsulate the parametric dependence of the gradient at saturation in

$$
\left|E_{z}^{(\text {sat })}\right| \simeq \frac{\left|E_{z}^{(\mathrm{sat}, \mathrm{max})}\right|}{\sqrt{1+\left[\gamma / \gamma_{c}\left(R_{\mathrm{ext}}\right)\right]^{4}}} .
$$

It is important to emphasize at this stage that the saturation is independent of the trigger bunch charge as the trigger bunch only initiates the stimulated emission process. Employing a low charge, low density beam, would still facilitate the maximal saturation possible, only at a different location $\mathrm{z}$ behind the bunch, i.e., longer saturation lengths. Figure 13 demonstrates the relation between saturation length $L_{\text {sat }}$ and the number of electrons in the trigger bunch $N_{\mathrm{tg}}$, for the operation point dimensions. The relation reads $L_{\text {sat }} \propto-\log \left(N_{\mathrm{tg}}\right)$, where evidently saturation lengths are at the $\mathrm{cm}$ scale.

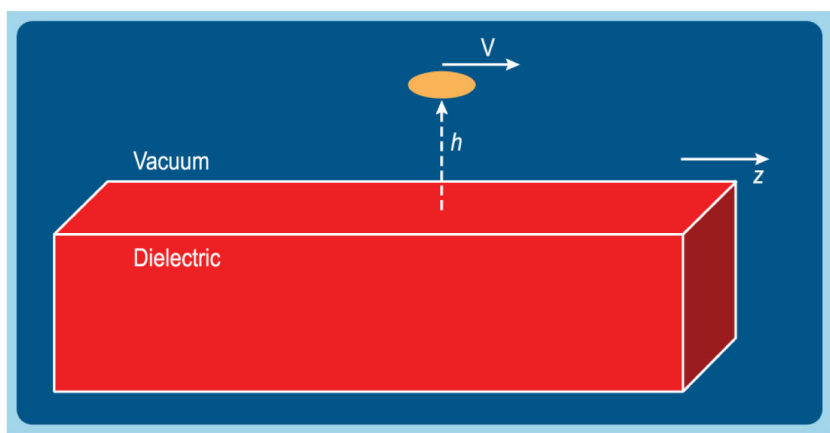

(a) Dielectric half space

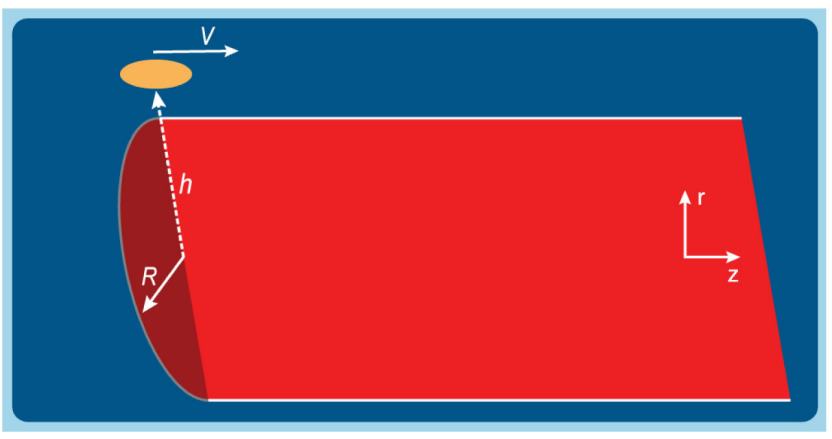

(b) Single dielectric rod

FIG. 14. A possible configuration for active medium acceleration can consist of a trigger electron bunch which propagates at a straight path with height $h$ above a dielectric half-space (a). For an analytic representation of the latter we can consider a bunch of electrons moving above a dielectric cylinder (b), where this design is equivalent to the half-space when taking the radius of curvature $R$ to infinity.

\section{PROPOSED EXPERIMENTAL SETUP}

To complete our study, we would like to consider possible setups for an active medium acceleration experiment. From the proof of principle perspective there are two conceivable configurations: in one case a bunch of electrons grazes above a dielectric half-space and for the analytic representation this can be reduced to a bunch of electrons moving on top of a dielectric cylinder; the general trend for the former can be obtained at the limit of infinite radius of curvature but maintaining the height of the bunch from the medium finite-see Fig. 14. In Appendix $\mathrm{C}$ we highlight the main results for this configuration, where the difficulty is a high degree of asymmetry. The latter results in a wake which consists of multiple azimuthal harmonics with multiple modes. Even in the ideal scenario of a single dominant mode for each harmonic, we find that several harmonics have similar contribution, which is very undesired in any acceleration scheme. Our analysis also shows that the trigger field strongly depends on the electron energy $\gamma$, hence higher energy trigger beams are necessary for short saturation lengths. Moreover, if the diameter of the electron beam is relatively large, then electrons traveling farthest from the surface will receive less energy, thereby creating a wide energy spread. 


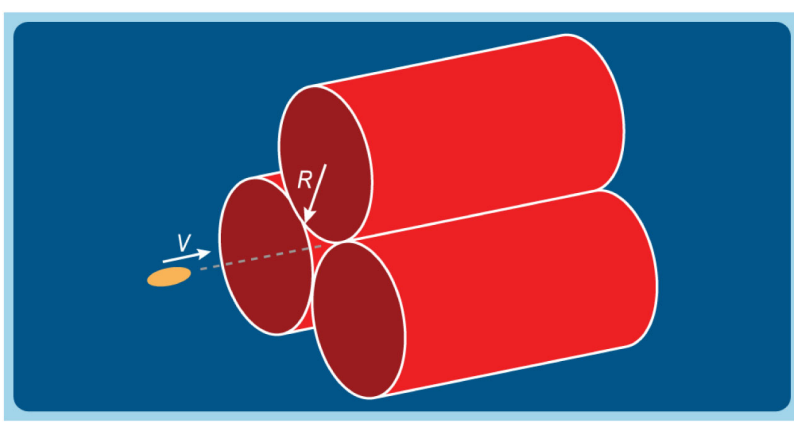

FIG. 15. Schematics of a structure which consists of three dielectric rods of identical radius $R$. The rods are attached to each other so that a bunch of electrons can be injected in the vacuum emerged between them. The model established in this study for a hollow rod can serve as an approximation for this configuration which is easier for assembly.

In order to improve the asymmetry we suggest a second configuration which is very simple for assembly and can be approximated by the analytic model described in this study. Rather than using a dielectric rod where a vacuum channel must be drilled inside it to allow for electron transport, we suggest a structure which consists of three dielectric rods of identical radius $R$ attached to each other whereby the bunch of electrons is launched in the emerging void between these three. To make the latter comparable with the analytic model, we consider the internal radius of the vacuum channel to determine a circle which is confined by three circles of radius $R$ representing the three solid state active rods, therefore, the effective internal radius is given by $R_{\text {int,eff }}=R(1 / \cos (\pi / 6)-1) \simeq 0.15 R$. The effective external radius is chosen such that, assuming uniform population inversion, the total energy stored is the same $\pi\left(R_{\text {ext,eff }}^{2}-R_{\text {int,eff }}^{2}\right)=3 \pi R^{2}$ - see Fig. 15 .

For an initial proof-of-principle experiment we consider the three rods design which would be relatively simple for implementation. With this configuration in mind, while considering the specifications available at ATF-BNL and Nd:YAG as the active medium, we listed in Table III the trigger bunch parameters and the optimal corresponding rod properties. Clearly, we take into account that the void created between the rods must be sufficient to confine the bunch. The predicted acceleration gradient at saturation for the effective geometry of the rods $\left(R_{\text {int,eff }}, R_{\text {ext,eff }}\right)$ would be $\simeq 10 \mathrm{MV} / \mathrm{m}$ according to Eq. (25) for a less than $5 \mathrm{~cm}$ long rod; the initial trigger field would be approximately $10^{-6} \mathrm{~V} / \mathrm{m}$ according to Eq. (21).

In order to achieve an acceleration gradient at the scale of $1 \mathrm{GV} / \mathrm{m}$ at saturation, we must use a structure which satisfies the operation point given in Sec. V, i.e., $R_{\mathrm{int}} \simeq 8 \mu \mathrm{m}, R_{\mathrm{ext}} \simeq 1 \mathrm{~mm}$; these dimensions are feasible for Nd-doped fibers [24]. The proper trigger beam radius (which must be smaller than $R_{\text {int }}$ ) is currently not feasible at ATF-BNL, yet the rest of the beam properties remain
TABLE III. Suggested set of parameters for a proof-of-principle experiment; Predicated $\sim 10 \mathrm{MeV}$ energy gain at saturation.

\begin{tabular}{lcc}
\hline \hline Parameter & Symbol & Value \\
\hline Trigger Bunch & $R_{\mathrm{tg}}[\mu \mathrm{m}]$ & \\
Radius & $\Delta_{\mathrm{tg}}[\mathrm{ps}]$ & 50 \\
Duration & $\gamma$ & 8 \\
Energy & $N_{\mathrm{tg}}$ & 100 \\
$\#$ of electrons & & $10^{4}$ \\
Rod & $R[\mathrm{~mm}]$ & \\
Radius & $R_{\text {int,eff }}[\mu \mathrm{m}]$ & 0.4 \\
Internal radius & $R_{\text {ext,eff }}[\mathrm{mm}]$ & $\sim 60$ \\
External radius & $L[\mathrm{~cm}]$ & $\sim 0.7$ \\
Length & & $\sim 5$ \\
\hline \hline
\end{tabular}

similar to those given in Table III and the rod length required to reach saturation may be smaller than $5 \mathrm{~cm}$.

Based on experimental data $[25,26]$ on (optical) damage threshold of silica, it has been predicted theoretically [27-29] that silica may withstand laser intensities which correspond to accelerating gradients between $1-10 \mathrm{GV} / \mathrm{m}$, according to pulse duration. We assume a similar threshold for active materials such as $\mathrm{Nd}$ :YAG.

We note that, as shown in Sec. V, for high energy trigger beams, the electromagnetic energy in the system escapes to the surrounding vacuum area, leading to a decreased gradient at saturation. This would demand the use of lower energy trigger beams, roughly below $\gamma=10^{4}$. Yet, for the relatively short length $L$ needed to achieve saturation, typically a few $\mathrm{cm}$, low energy beams can accelerate high energy beams. This is possible as the phase slip $(\omega L / c)\left(\beta_{\mathrm{tg}}{ }^{-1}-\beta_{\mathrm{tr}}{ }^{-1}\right)$ between a trigger beam with $\gamma_{\mathrm{tg}}=10^{3}$, for instance, and a trailing beam with $\gamma_{\mathrm{tr}}=10^{5}$, is expected to be roughly $0.05 \pi$. Moreover, in order to avoid this energy spread outside the dielectric, a confining Bragg structure can be employed, as discussed in [13].

From a practical perspective, the effect of possible microprotrusions on the microbunch and wake should be considered. The latter were investigated in the past [30] by modeling them as an ensemble of grooves of random geometry in a closed metallic structure. This model facilitates to determine analytic expressions for the average energy emitted per groove and its standard deviation. For a relativistic bunch, both quantities are shown to be virtually independent of the momentum. Assuming a microprotrusion with a characteristic of average size $\langle g\rangle$ and standard deviation $\Delta g$, the standard deviation of energy emitted per groove by an electron $q_{\mathrm{el}}$ is given by $\Delta W / N \simeq\left(q_{\mathrm{el}}^{2} / 4 \pi \epsilon_{0} R_{\text {int }}\right) \times 0.3\left(\Delta g / R_{\text {int }}\right)^{1 / 4}$. This can provide us with a measure of the roughness effect on the trigger bunch. As an order of magnitude, current technology can ensure roughness of less than $10 \mathrm{~nm}$ [31], hence the noise induced in the trigger bunch by one microprotrusion is of the order of $4.3 \times 10^{-7} \mathrm{eV}\left(R_{\text {int }} \simeq 100 \mu \mathrm{m}\right)$. If we assume that 
the spacing between two microprotrusions is on the order of 10 times their size, in a $10 \mathrm{~cm}$ long structure there are $10^{6}$ micro-protrusions inducing $0.43 \mathrm{eV}$ in the trigger bunch. For a relativistic $(\gamma=100)$ trigger bunch, the intrinsic energy spread is typically on the order of $0.1 \%$ which is 5 orders of magnitude larger than that induced by the microprotrusions.

\section{SUMMARY}

In this study, a quasianalytic model was established for a two-beam acceleration configuration based on Cherenkov wakefield amplification by an active medium. The structure consists of a solid-state hollow rod with a vacuum channel in its core, facilitating relativistic electron transport without scattering. The Cherenkov wake, generated by the trigger bunch, contains a large and discrete number of eigenmodes, each corresponds to a different eigenfrequency. The mode with eigenfrequency closest to the resonance of the active medium is shown to exponentially grow in space and in time. The energy source of this growing mode is the energy stored in the medium. Sufficiently away from the trigger bunch, the medium is depleted and the gain virtually vanishes, thus the system reaches saturation.

The paradigm is described by three quantities: the longitudinal electric field immediately after the trigger bunch, the exponential gain and the saturation. The main results of this study are the scaling laws which determine the dependence of these three quantities on the electric and geometric parameters as given in Eqs. (21), (22), (26).

In addition, we suggest an optimal set of parameters for a future proof of principle experiment. According to our numerical estimates, the proposed configuration facilitates an accelerating gradient at saturation of approximately $1 \mathrm{GV} / \mathrm{m}$ for a few $\mathrm{cm}$ long rod, with a vacuum channel of $8 \mu \mathrm{m}$ and external radius of $1 \mathrm{~mm}$.

\section{ACKNOWLEDGMENTS}

This study was supported by Israel Science Foundation. We wish to thank Dr. Wayne D. Kimura for his valuable comments.

\section{APPENDIX A: LINEAR MODEL-SYMBOLS DICTIONARY}

This Appendix contains definitions of several expressions excluded from Sec. II for brevity.

The functions defining $E_{z}^{(\mathrm{sec})}(r, \tau)$ in Eq. (5) are given by

$$
\begin{aligned}
& a_{11}(\omega) \equiv 1+\zeta^{2} \frac{K_{1}\left(\Gamma R_{\mathrm{int}}\right)}{K_{0}\left(\Gamma R_{\mathrm{int}}\right)} \frac{K_{1}\left(\Gamma R_{\mathrm{ext}}\right)}{K_{0}\left(\Gamma R_{\mathrm{ext}}\right)} \\
& a_{12}(\omega) \equiv \zeta\left[\frac{K_{1}\left(\Gamma R_{\mathrm{ext}}\right)}{K_{0}\left(\Gamma R_{\mathrm{ext}}\right)}-\frac{K_{1}\left(\Gamma R_{\mathrm{int}}\right)}{K_{0}\left(\Gamma R_{\mathrm{int}}\right)}\right] \\
& a_{21}(\omega) \equiv 1-\zeta^{2} \frac{I_{1}\left(\Gamma R_{\mathrm{int}}\right)}{I_{0}\left(\Gamma R_{\mathrm{int}}\right)} \frac{K_{1}\left(\Gamma R_{\mathrm{ext}}\right)}{K_{0}\left(\Gamma R_{\mathrm{ext}}\right)} \\
& a_{22}(\omega) \equiv \zeta\left[\frac{I_{1}\left(\Gamma R_{\mathrm{int}}\right)}{I_{0}\left(\Gamma R_{\mathrm{int}}\right)}+\frac{K_{1}\left(\Gamma R_{\mathrm{ext}}\right)}{K_{0}\left(\Gamma R_{\mathrm{ext}}\right)}\right]
\end{aligned}
$$

where $\zeta \equiv \gamma \beta \sqrt{\varepsilon-\beta^{-2}} / \varepsilon$.

The longitudinal wake induced by a single charged loop, as given in Eq. (12) contains the following expression

$$
\begin{aligned}
\bar{a}\left(\omega_{0, n}\right)= & -2 \frac{\left|\omega_{0, n}\right|}{c}\left(R_{\mathrm{ext}}-R_{\mathrm{int}}\right) \\
& \times \frac{\left(a_{12} / a_{11}\right)-\left(a_{22} / a_{21}\right)}{\sqrt{\left(a_{12} / a_{11}\right)^{2}+1} \sqrt{\left(a_{22} / a_{21}\right)^{2}+1}} \frac{\sqrt{a_{11}^{2}+a_{12}^{2}}}{\sqrt{a_{21}^{2}+a_{22}^{2}}}
\end{aligned}
$$

where $a_{11} \equiv a_{11}\left(\omega_{0, n}\right), a_{12} \equiv a_{12}\left(\omega_{0, n}\right), a_{21} \equiv a_{21}\left(\omega_{0, n}\right)$ and $a_{22} \equiv a_{22}\left(\omega_{0, n}\right)$, as defined in Eq. (A1). Moreover, the amplitudes of the four different modes introduced in Eq. (12) read

$$
b_{n, i}=\frac{\left(s_{n, i}\right)^{2}+\left(s_{n, i}\right) \Delta \omega+\omega_{\mathrm{res}}^{2}}{\sum_{l \neq i}\left(s_{n, i}-s_{n, l}\right)}
$$

\section{APPENDIX B: FIELD COMPONENTS IN FREQUENCY DOMAIN}

In this Appendix we define all field components in the frequency domain induced by a single charged loop traversing our configuration of which consist of a hollow active medium rod with a vacuum channel in its center. The longitudinal wake is given by

$$
E_{z}^{(\mathrm{sec})}(r, z, \omega)=-\frac{q \mu_{0}}{(2 \pi)^{2}} \exp \left[-j \omega\left(\frac{z-z_{\mathrm{so}}}{c \beta}\right)\right] \times \begin{cases}j \omega\left(\frac{1}{\beta^{2}}-1\right) A(\omega) I_{0}(\Gamma r) & 0 \leq r<\mathrm{R}_{\mathrm{int}} \\ j \omega\left(\frac{1}{\beta^{2} \varepsilon_{r}}-1\right) B(\omega) J_{0}(\Lambda r)+j \omega\left(\frac{1}{\beta^{2} \varepsilon_{r}}-1\right) C(\omega) Y_{0}(\Lambda r) & R_{\mathrm{int}}<r<R_{\mathrm{ext}} \\ j \omega\left(\frac{1}{\beta^{2}}-1\right) D(\omega) K_{0}(\Gamma r) & R_{\mathrm{ext}} \leq r<\infty\end{cases}
$$


The radial field component reads

$$
E_{r}^{(\mathrm{sec})}(r, z, \omega)=\frac{c}{\beta} \frac{q \mu_{0}}{(2 \pi)^{2}} \exp \left[-j \omega\left(\frac{z-z_{\mathrm{so}}}{c \beta}\right)\right] \times\left\{\begin{array}{cc}
A(\omega) \Gamma I_{1}(\Gamma r) & 0 \leq r<\mathrm{R}_{\mathrm{int}} \\
-\frac{1}{\varepsilon_{r}} B(\omega) \Lambda J_{1}(\Lambda r) & R_{\mathrm{int}}<r<R_{\mathrm{ext}} \\
-\frac{1}{\varepsilon_{r}} C(\omega) \Lambda Y_{1}(\Lambda r) & \\
-D(\omega) \Gamma K_{1}(\Gamma r) & R_{\mathrm{ext}} \leq r<\infty
\end{array}\right.
$$

The azimuthal magnetic field is given by

$$
H_{\phi}^{(\mathrm{sec})}=-\frac{q}{(2 \pi)^{2}} \exp \left[-j \omega\left(\frac{z-z_{\mathrm{so}}}{c \beta}\right)\right] \times\left\{\begin{array}{cc}
-A(\omega) \Gamma I_{1}(\Gamma r) & 0 \leq r<\mathrm{R}_{\mathrm{int}} \\
B(\omega) \Lambda J_{1}(\Lambda r)+C(\omega) \Lambda Y_{1}(\Lambda r) & R_{\mathrm{int}}<r<R_{\mathrm{ext}} \\
D(\omega) \Gamma K_{1}(\Gamma r) & R_{\mathrm{ext}} \leq r<\infty
\end{array}\right.
$$

where the remaining field components $H_{z}{ }^{(\mathrm{sec})}, E_{\phi}{ }^{(\mathrm{sec})}$, $H_{r}{ }^{(\mathrm{sec})}$, are zero.

\section{APPENDIX C: SINGLE ROD CONFIGURATION}

It is the purpose of this Appendix to determine the wakefield generated by a single electron or an electron bunch injected parallel to an active medium rod, e.g., $\mathrm{Nd}$ : YAG, as illustrated in Fig. 14(b). We define $R$ as the rod radius, $r_{\mathrm{so}}$ as the source radial location and $\phi_{\mathrm{so}}$ as the azimuthal coordinate. To establish the model we follow similar steps as in the hollow rod configuration, given in Sec. II.

\section{Linear model}

For the following analysis we define the normalized quantities: $\Omega \equiv \omega R / c$ is the normalized frequency, $\Omega_{\mathrm{p}} \equiv$ $\omega_{\mathrm{pl}} R / c$ is the normalized plasma frequency, $\Omega_{\mathrm{res}}$ is the normalized medium resonance frequency and $\Delta \Omega$ is the normalized bandwidth. The frequency dependent dielectric coefficient of the active medium rod is given in Eq. (1). The current density, $J_{z}(r, \phi, z ; \omega)$, excited by a single electron moving in a general cylindrical coordinate system (when no azimuthal symmetry is considered) is

$$
J_{z}=-\frac{q_{\mathrm{el}}}{2 \pi r} \delta\left(r-r_{\mathrm{so}}\right) \delta\left(\phi-\phi_{\mathrm{so}}\right) \exp \left(-j \frac{\omega}{\beta c} z\right)
$$

where $\beta c=V$ is the electron velocity. With this current density we can determine the Green's function of the configuration.

\section{a. Green's function}

In order to determine the wakefield generated by a single electron travelling above the surface of the rod, we evaluate the longitudinal magnetic vector potential induced by the electron current density. The magnetic vector potential is a superposition of two components. The first is dictated by nonhomogeneous solution, i.e., the primary one which is determined by the current density ignoring the presence of the rod, $A_{z}{ }^{\text {(pri) }}(r, \phi, z ; \omega)$; in what follows we omit the explicit expression for the variables. The second is the homogeneous solution, i.e., the secondary contribution and it accounts for the presence of the rod alone. The superposition of the two satisfies the boundary conditions at $r=R$. First, in the absence of the dielectric cylinder

$$
\begin{aligned}
A_{z}{ }^{(\mathrm{pri})}= & -\frac{q \mu_{0}}{(2 \pi)^{2}} e^{-j \bar{\omega}_{\bar{V}} z} \sum_{\nu=-\infty}^{\infty} e^{j \nu\left(\phi-\phi_{\mathrm{so}}\right)} \\
& \times \begin{cases}I_{\nu}\left(\Gamma r_{\mathrm{so}}\right) K_{\nu}(\Gamma r) & r>r_{\mathrm{so}} \\
K_{\nu}\left(\Gamma r_{\mathrm{so}}\right) I_{\nu}(\Gamma r) & r<r_{\mathrm{so}}\end{cases}
\end{aligned}
$$

where $I_{\nu}, K_{\nu}$ are the modified Bessel functions of the first and second kind respectively and $\nu \in \mathbb{N}$ are the discrete azimuthal harmonics describing the magnetic vector potential $\left(c^{2} \Gamma^{2} \equiv \omega^{2} / \beta^{2} \gamma^{2}\right.$ as defined in the main text). Applying Lorentz gauge, the primary magnetic vector potential dictates the longitudinal electric field

$$
\begin{aligned}
E_{z}^{(\mathrm{pri})}= & -\frac{j \omega}{\gamma^{2} \beta^{2}} \frac{q \mu_{0}}{(2 \pi)^{2}} e^{-j{ }_{V} z} \sum_{\nu=-\infty}^{\infty} e^{j \nu\left(\phi-\phi_{\mathrm{so}}\right)} \\
& \times \begin{cases}I_{\nu}\left(\Gamma r_{\mathrm{so}}\right) K_{\nu}(\Gamma r) & r>r_{\mathrm{so}} \\
K_{\nu}\left(\Gamma r_{\mathrm{so}}\right) I_{\nu}(\Gamma r) & r<r_{\mathrm{so}}\end{cases}
\end{aligned}
$$

The primary tangential field component $E_{\phi}^{(\mathrm{pri})}, H_{\phi}^{(\mathrm{pri})}$ can also be determined by the primary magnetic vector potential, while the longitudinal magnetic field $H_{z}^{(\text {pri) }}=0$. Next, we account for the secondary homogeneous solution in the presence of the cylinder and absence of sources which dictates the longitudinal field components

$E_{z}^{(\mathrm{sec})}=e^{-j \frac{\omega}{V} z} \sum_{\nu=-\infty}^{\infty} e^{-j \nu\left(\phi-\phi_{\mathrm{so}}\right)} \begin{cases}A_{\nu} K_{\nu}(\Gamma r) & r>R \\ B_{\nu} J_{\nu}(\Lambda r) & r<R\end{cases}$ 
$H_{z}^{(\mathrm{sec})}=e^{-j \bar{T}_{z}} \sum_{\nu=-\infty}^{\infty} e^{-j \nu\left(\phi-\phi_{\mathrm{so}}\right)} \begin{cases}C_{\nu} K_{\nu}(\Gamma r) & r>R \\ D_{\nu} J_{\nu}(\Lambda r) & r<R\end{cases}$

where $A_{\nu}, B_{\nu}, C_{\nu}, D_{\nu}$ are unknown amplitudes. The azimuthal fields $E_{\phi}^{(\mathrm{sec})}, H_{\phi}^{(\mathrm{sec})}$ may be deduced by the longitudinal components. The continuity of the four tangential components $E_{z}, H_{z}, E_{\phi}, H_{\phi}$ at the boundary $r=R$ imposes four boundary conditions which determine the amplitudes of the corresponding fields. For the longitudinal electric field we find the amplitudes

$$
\begin{aligned}
& A_{\nu}=-\frac{j \omega a_{\nu}}{\dot{K}_{\nu}{ }^{2}(\Gamma R)} \frac{\mathcal{N}_{\nu}(\Omega)}{\mathcal{D}_{\nu}(\Omega)} \\
& B_{\nu}=\frac{1}{J_{\nu}(\Lambda R)}\left[\frac{j \omega a_{\nu}}{\gamma^{2} \beta^{2}} I_{\nu}(\Gamma R)+A_{\nu} K_{\nu}(\Gamma R)\right]
\end{aligned}
$$

where we define $a_{\nu}=-q \mu_{0} K_{\nu}\left(\Gamma r_{\mathrm{so}}\right) /(2 \pi)^{2}$ and the full expressions for $\mathcal{N}_{\nu}, \mathcal{D}_{\nu}$ read

$$
\begin{aligned}
\mathcal{N}_{\nu}(\Omega)= & \frac{\nu^{2} \gamma^{2}}{\Omega^{2}}\left(1+\frac{1}{\bar{\varepsilon} \gamma^{2} \beta^{2}}\right)^{2} J_{\nu}{ }^{2} K_{\nu} I_{\nu} \\
& -\dot{K}_{\nu} \dot{I}_{\nu}\left(J_{\nu}+\frac{\varepsilon_{r}}{\gamma \beta \sqrt{\bar{\varepsilon}}} \dot{J}_{\nu} \frac{I_{\nu}}{\dot{I}_{\nu}}\right)\left(J_{\nu}+\frac{1}{\gamma \beta \sqrt{\bar{\varepsilon}}} \frac{K_{\nu}}{\dot{K}_{\nu}} \dot{J}_{\nu}\right)
\end{aligned}
$$

$$
\begin{aligned}
& \mathcal{D}_{\nu}(\Omega)=\gamma^{2} \beta^{2}\left(\frac{\nu \gamma}{\Omega}\right)^{2}\left(1+\frac{1}{\bar{\varepsilon} \gamma^{2} \beta^{2}}\right)^{2} J_{\nu}{ }^{2} \frac{K_{\nu}{ }^{2}}{\dot{K}_{\nu}{ }^{2}}
\end{aligned}
$$

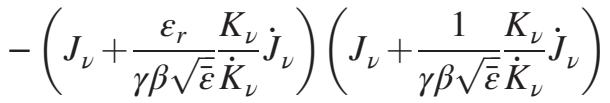

where $\bar{\varepsilon}=\varepsilon_{r}-\beta^{-2}$. Here we defined $K_{\nu} \equiv K_{\nu}(\Gamma R)$, $I_{\nu} \equiv I_{\nu}(\Gamma R), \quad J_{\nu} \equiv J_{\nu}(\Omega \sqrt{\bar{\varepsilon}})$. Additionally, $\dot{J}_{\nu}$ and $\dot{K}_{\nu}$ represent the derivative of $J_{\nu}(x)$ and $K_{\nu}(x)$ with respect to their argument $x$, respectively. Using the above expressions, we can write the time domain longitudinal electric field imposed by a single electron

$$
\begin{aligned}
E_{z}{ }^{(\mathrm{sec})}\left(r, \phi, \tau^{\prime}\right)= & \frac{q_{\mathrm{el}}}{4 \pi^{2} R^{2} \varepsilon_{0}} \frac{\partial}{\partial \tau^{\prime}} \sum_{\nu=-\infty}^{\infty} \exp \left[j \nu\left(\phi-\phi_{\mathrm{so}}\right)\right] \\
& \times \int_{-\infty}^{\infty} d \Omega \frac{K_{\nu}\left(\Gamma r_{\mathrm{so}}\right) K_{\nu}(\Gamma r)}{\dot{K}_{\nu}{ }^{2}} \frac{\mathcal{N}_{\nu}(\Omega, \varepsilon)}{\mathcal{D}_{\nu}(\Omega, \varepsilon)} \\
& \times \exp \left(j \Omega \tau^{\prime}\right)
\end{aligned}
$$

where $\tau^{\prime}=(c / R)(t-z / V)$. We can now employ Cauchy's residue theorem to evaluate the integral based on the poles of the integrand; these are given by the poles of $\mathcal{N}_{\nu} / \mathcal{D}_{\nu}$. Around these poles, considering high energies $\gamma \gg 1$, the latter is approximated as

$$
\frac{\mathcal{N}_{\nu}(\Omega)}{\mathcal{D}_{\nu}(\Omega)} \simeq-\frac{1}{\gamma^{2}} \frac{\varepsilon_{r}}{\sqrt{\bar{\varepsilon}}} \frac{\dot{J}_{\nu}(\Omega \sqrt{\bar{\varepsilon}}) / \Omega}{J_{\nu}(\Omega \sqrt{\bar{\varepsilon}})}
$$

The poles are clearly determined by the zeros of the Bessel function $J_{\nu}(\Omega \sqrt{\bar{\varepsilon}}) \equiv J_{\nu}\left(p_{s}\right)=0$, where $s=1,2 \ldots \infty$. We can now establish the zero order resonances $\Omega_{0, \nu, s}$ for the passive case $\delta \varepsilon=0$, where $\nu$ are the azimuthal harmonics and $s$ represent the discrete modes of each harmonic; Next we account for $\delta \varepsilon \neq 0$ and establish the frequency deviation due to this slippage. We expand the denominator in both variables $\left(\Omega_{0, \nu, s}, \varepsilon\right)$ up to first order in the same manner as in Eq. (8); This expansion simplifies Eq. (C9) to the form of Eq. (9). The corresponding Green's function reads

$$
\begin{aligned}
G_{\nu, s}\left(\tau^{\prime}\right) & =\frac{1}{2} \pi \int_{-\infty}^{\infty} d \Omega \exp \left(j \Omega \tau^{\prime}\right) \frac{\left(-\Omega^{2}+j \Omega \Delta \Omega+\Omega_{\mathrm{res}}^{2}\right)}{\mathcal{D}_{G, \nu, s}(\Omega)} \\
\mathcal{D}_{G, \nu, s}(\Omega) & =\left(\Omega^{2}-\Omega_{0, \nu, s}^{2}\right)\left(-\Omega^{2}+j \Omega \Delta \Omega+\Omega_{\mathrm{res}}^{2}\right)-\bar{\Omega}_{\mathrm{p}, \nu, \mathrm{s}}^{4}
\end{aligned}
$$

where we define the normalized plasma frequency $\Omega_{\mathrm{p}, \nu, \mathrm{s}}^{4} \equiv \Omega_{\mathrm{p}}^{2} \mathcal{D}_{\nu, \varepsilon} / \mathcal{D}_{\nu, \Omega^{2}} \simeq \Omega_{\mathrm{p}}^{2} \Omega_{0, \nu, s}^{2} / \bar{\varepsilon}_{r}, \mathcal{D}_{\nu, \Omega^{2}}$ is the derivative with respect to $\Omega^{2}$ and $\mathcal{D}_{\nu, \varepsilon}$ is the derivative with respect to $\varepsilon$. The denominator is again a fourth order polynomial in $j \Omega$, where we mark its four poles by $g_{\nu, s, i}$ (each $\Omega_{0, \nu, s}$ gives four solutions indexed by i). We solve the Green's function integral to reach an analytic expression for the time domain longitudinal electric field

$$
\begin{aligned}
E_{z}(\mathrm{sec})\left(r, \phi, z, \tau^{\prime}\right) \simeq & E_{1}^{(\mathrm{sec})} \sum_{\nu=-\infty}^{\infty} \sum_{s=1}^{\infty} e^{j \nu\left(\phi-\phi_{\mathrm{so}}\right)} \\
& \times \frac{K_{\nu}\left(\Gamma_{\nu, s} r_{\mathrm{so}}\right) K_{\nu}\left(\Gamma_{\nu, s} r\right)}{\dot{K}_{\nu}{ }^{2}} \\
& \times \sum_{i=1}^{4} b_{\nu, s, i}\left[\delta\left(\tau^{\prime}\right)+g_{\nu, s, i} h\left(\tau^{\prime}\right) e^{g_{\nu, s, i} \tau^{\prime}}\right] \\
E_{1}^{(\mathrm{sec})} \equiv & 4 \varepsilon_{r} q / 4 \pi R^{2} \varepsilon_{0} \gamma^{2} \bar{\varepsilon}
\end{aligned}
$$

where $h\left(\tau^{\prime}\right)$ is the step function and the amplitudes $b_{\nu, s, i}$ are given by

$$
b_{\nu, s, i}=\frac{g_{\nu, s, i}^{2}+\Delta \Omega\left(g_{\nu, s, i}\right)+\Omega_{\mathrm{res}}^{2}}{\sum_{l \neq i} g_{\nu, s, i}-g_{\nu, s, l}}
$$

The zeros around the resonance frequency are of the form $g_{\nu, s, i}= \pm j \Omega_{\mathrm{res}} \pm \delta \Omega$. One of the four solutions indexed by $\mathrm{i}$ for each $\Omega_{\nu, s}$ may be a growing mode, with $\delta \Omega_{\nu, s, i}>0$. Each harmonic $\nu_{0}$ may have several solutions which satisfy $\Omega_{0, \nu_{0}, s} \simeq \Omega_{\mathrm{res}}$ and $\delta \Omega>0$. Hence, there are multiple interacting harmonics $\nu$ with multiple interacting modes $s$; this is a direct outcome of the configuration azimuthal asymmetry. The ideal and mandatory scenario for an accelerator scheme is of a single harmonic with a single dominant 
mode, extracting the maximum energy from the medium in a single-pass amplification.

\section{b. Electron Bunch}

We proceed to evaluate the wake of a finite-size electron bunch. First, we consider a circular electron bunch with uniform distribution in the radial, longitudinal, and azimuthal directions. In order to account for the field generated by each electron in the trigger bunch, we integrate over all sources. The longitudinal electric field is thus given by

$$
\begin{aligned}
\left\langle E_{z}^{(\mathrm{sec})}\left(r, \phi ; \tau^{\prime}\right)\right\rangle \simeq & N_{\mathrm{tg}} E_{1}^{(\mathrm{sec})} \sum_{\nu=-\infty}^{\infty} \sum_{s=1}^{\infty} e^{j \nu \phi}\left\langle e^{-j \nu \phi_{k}}\right\rangle_{k} \\
& \times \frac{K_{\nu}\left(\Gamma_{\nu, s} r\right)}{\dot{K}_{\nu}{ }^{2}}\left\langle K_{\nu}\left(\Gamma_{\nu, s} r_{k}\right)\right\rangle_{k} \\
& \times \sum_{i=1}^{4} b_{\nu, s, i} g_{\nu, s, i} \exp \left(g_{\nu, s, i} \tau^{\prime}\right) \\
& \times \operatorname{sinch}\left[\frac{R}{c} \frac{\Delta_{\mathrm{tg}}}{2} g_{\nu, s, i}\right]
\end{aligned}
$$

where $k$ is the index for each electron in the trigger bunch and we exploit the equivalency $N^{-1} \sum_{k} \equiv\langle\ldots\rangle_{k}$ when summing the sources. A second option, is employing an arch shaped bunch, surrounding the rod with azimuthal range $\Delta_{\phi}$. This form excites a field given by

$$
\begin{aligned}
\left\langle\tilde{E}_{z}^{(\mathrm{sec})}\left(r, \phi ; \tau^{\prime}\right)\right\rangle & \simeq N_{\mathrm{tg}} E_{1}^{(\mathrm{sec})} \sum_{\nu=-\infty}^{\infty} \sum_{s=1}^{\infty} e^{j \nu \phi} \operatorname{sinc}\left(\nu \frac{\Delta_{\phi}}{2}\right) \\
& \times \frac{K_{\nu}\left(\Gamma_{\nu, s} r\right)}{\dot{K}_{\nu}^{2}} \frac{1}{h \Delta_{r}} \int_{h-\Delta_{r} / 2}^{h+\Delta_{r} / 2} d r_{\mathrm{so}} r_{\mathrm{so}} K_{\nu}\left(\Gamma_{\nu, s} r_{\mathrm{so}}\right) \\
& \times \sum_{i=1}^{4} b_{\nu, s, i} g_{\nu, s, i} \exp \left(g_{\nu, s, i} \tau^{\prime}\right) \\
& \times \operatorname{sinch}\left[\frac{R}{c} \frac{\Delta_{\mathrm{tg}}}{2} g_{\nu, s, i}\right]
\end{aligned}
$$

where $h$ is the radial center of the beam above the central axis of $\operatorname{rod}, \Delta_{r}$ is the radial length and $\operatorname{sinch}(x) \equiv$ $\sinh (x) / x$. In order to achieve an ideal state of a single dominant harmonic, the arch shaped bunch is preferable, as the angular distribution is determined by $F(\phi)=1 / \Delta_{\phi}$ for $-\pi / 2<\phi<\pi / 2$ and 0 otherwise. The discrete Fourier transform for the given distribution, considering $\Delta_{\phi}=\pi$, is $f(\nu)=\sin (\nu \pi / 2) / \pi^{2} \nu$. Therefore, the dominant harmonic is zero, while the energy of each odd harmonic decreases by a square factor, and the even modes have zero contribution.

\section{Trigger field}

The trigger field marked $\left|E_{z}^{(0)}\right|$ measured at the tail of the trigger bunch $\left(\Delta_{\mathrm{tg}} / 2\right)$, is determined by Eq. (C14) and averaged over the radial cross section of the vacuum

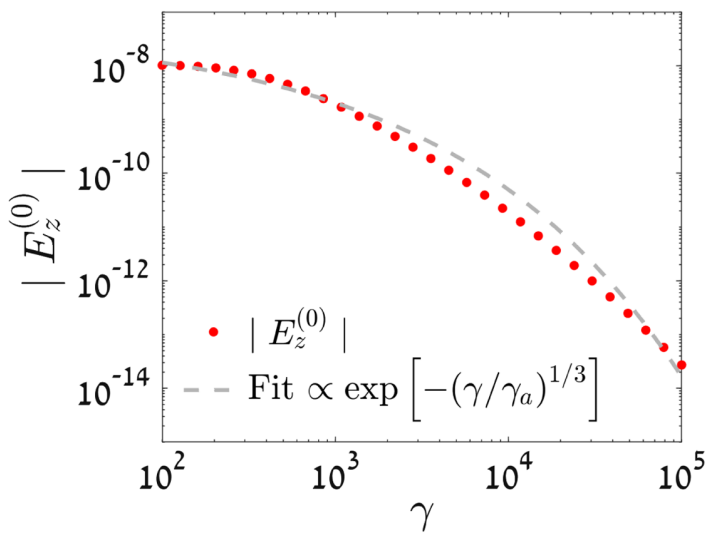

(a)

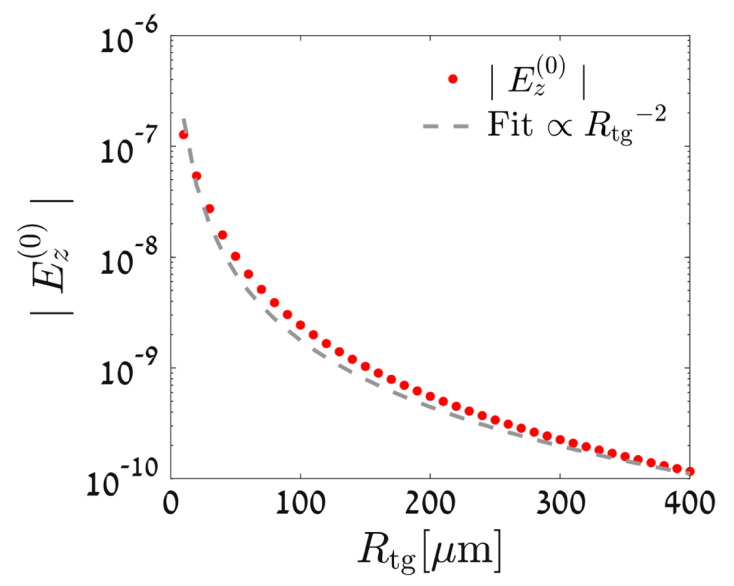

(b)

FIG. 16. Trigger field as a function of the trigger beam energy $\gamma$ (a) and radius $R_{\mathrm{tg}}$ (b).

channel. In this section we discuss the trigger field generated by the dominant mode of the zero harmonic and examine its dependence on the geometry of the configuration and the trigger electron energy $\gamma$ for $N_{\text {tg }}=10^{4}$. Figure 16(a) shows the trigger field as a function of $\gamma$ for $R=2 \mathrm{~mm}$ and $R_{\mathrm{tg}}=50 \mu \mathrm{m}$. The curve indicates a relation of $\propto \exp \left[-\left(\gamma / \gamma_{a}\right)^{1 / 3}\right]$ where $\gamma_{a} \simeq 30$.

According to Fig. 16(b) we find the dependency of the trigger field on $R_{\mathrm{tg}}$ scales as $\left|E_{z}^{(0)}\right| \propto R_{\mathrm{tg}}{ }^{-2}$, where we take $R=2 \mathrm{~mm}, \gamma=100$ and consider $h=R+R_{\mathrm{tg}}$. The overall relation thus reads

$$
\left|E_{z}{ }^{(0)}\right| \simeq 4 \times 10^{-8}[\mathrm{~V} / \mathrm{m}]\left(\frac{50[\mu \mathrm{m}]}{R_{\mathrm{tg}}}\right)^{2} \exp \left[-\left(\gamma / \gamma_{a}\right)^{1 / 3}\right]
$$

\section{Growing harmonics and modes}

The longitudinal wake has multiple harmonics and modes as clearly evident in Eq. (C11). In this section we examine the contribution of harmonics $\nu$ and growing 


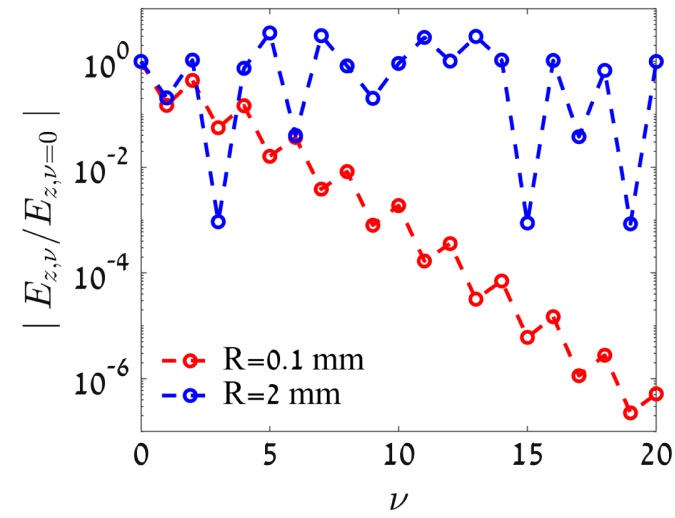

FIG. 17. The Longitudinal electric field generated by a single electron for each harmonic, normalized by the magnitude for the zero harmonic. The curves represent $R=2 \mathrm{~mm}$ (blue) and $R=$ $0.1 \mathrm{~mm}$ (red) for the case where $\gamma=100$.

modes $s$ of the configuration to the wake, keeping in mind that the best case scenario for an accelerator paradigm is when a single mode of a single harmonic extracts the maximum energy from the medium. In the following we consider the longitudinal field determined by Eq. (C11) for a single electron source located at a height of $50 \mu \mathrm{m}$ above the dielectric rod, where the rod parameters correspond to $\mathrm{Nd}: Y A G$. The field is calculated at a point $z \simeq 6 \mathrm{~cm}$ behind the trigger electron, which has an energy of $\gamma=100$.

\section{a. Harmonics and modes contribution}

We first analyze the relative contribution of the different harmonics. Figure 17 shows the normalized longitudinal field magnitude for each harmonic in the range $\nu=[0,20]$ for $R=2 \mathrm{~mm}$ and $R=0.1 \mathrm{~mm}$. Each harmonic is evaluated at the maximal growing mode indicated by $\delta \Omega_{\nu, s_{0}, i_{0}}$. Evidently, the radius of the active medium has a significant influence on the number of interacting harmonics. For $R=0.1 \mathrm{~mm}$ the zero harmonic is dominant. Yet, there are roughly four significant harmonics while the magnitude rapidly decreases for larger harmonics. For $R=2 \mathrm{~mm}$, there are more than 15 harmonics with similar contribution, implying that multiple harmonics (with multiple growing modes) may interact with the medium and interfere with one another. The behavior remains similar for different electron height values above the rod.

Second, Fig. 18 shows the longitudinal field of the zero harmonic for several growing modes $\bar{s}$ around the dominant mode with highest amplitude; i.e., $\bar{s}=s_{0}+\delta s$; their magnitude is normalized by the field amplitude for $s_{0}$. In the case where $R=0.1 \mathrm{~mm}$, we clearly see a single dominant mode, where for $R=2 \mathrm{~mm}$, there are two growing modes with similar gain.

We can conclude that an efficient configuration would require an active medium rod with a radius at the scale of $R \simeq 0.1 \mathrm{~mm}$.

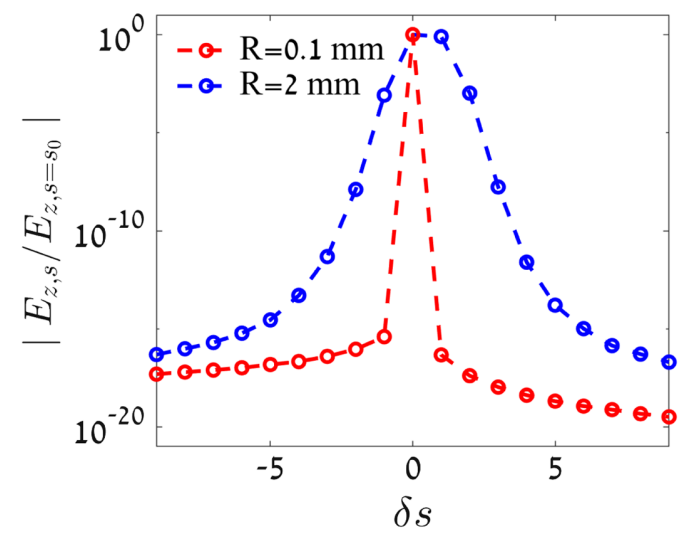

FIG. 18. Different modes of the longitudinal electric field induced by the zero harmonic of a single trigger electron. The field is normalized by the dominant mode with maximal amplitude. Curves represent $R=2 \mathrm{~mm}$ (blue) and $R=$ $0.1 \mathrm{~mm}$ (red) for $\gamma=100$.

\section{b. Exponential growth}

The exponential gain parameter is determined by the roots of $\mathcal{D}_{G, \nu, s}(\Omega)$ (Eq. $\left.(\mathrm{C} 10)\right)$. These roots are determined by the active medium parameters alone, thus when considering Nd:YAG, the gain parameter remains at the scale of $\operatorname{Re}\left(g_{\nu, s_{0}, i_{0}}\right) \simeq 1$ for the dominant harmonic, where the exponential gain is determined by $\operatorname{Re}\left(g_{\nu, s_{0}, i_{0}} c / R\right)$.

\section{Saturation assessment}

The longitudinal electric field growth given by the linear model is limited by the maximal energy that may be extracted from the medium. In order to evaluate the field at saturation $\left|E_{z}^{(\text {sat })}\right|$, we determine the interaction dielectric coefficient according to the same process described in the main text, leading to Eq. (24). First, we consider the beam parameters achievable at BNL, Table II and in the following we limit our discussion to a rod radius of $R=0.1 \mathrm{~mm}$, supporting the optimal case of a single dominant mode. We analyze the gradient at saturation assuming an ideal scenario of a single interacting harmonic $\nu_{0}$ which satisfies $\Omega_{0, \nu_{0}} \simeq \Omega_{\text {res }}$, hence the following results may serve as an upper limit approximation. For the given parameters, the gradient at saturation is on the scale of $E_{z}^{\text {(sat) }} \simeq 50 \mathrm{MV} / \mathrm{m}$, where the maximal gradient is at the scale of $0.1 \mathrm{GV} / \mathrm{m}$, achievable for $R_{\text {tr }}<10 \mu \mathrm{m}$. The most significant parameters dictating the gradient magnitude at saturation is the trailing bunch radius $R_{\text {tr }}$ and the trigger bunch energy $\gamma$.

Figure 19(a) shows the dependence of the field at saturation on $R_{\mathrm{tr}}$, where the height of the bunch above the rod is relative to the beam radius, i.e., $h=R+R_{\mathrm{tr}}$ for $R=0.1 \mathrm{~mm}$. The relation reads $\left|E_{z}^{(\mathrm{sat})}\right| \propto R_{\mathrm{tr}}{ }^{-2 / 3}$. As evident, the maximal gradient is at the scale of $\left|E_{z}^{(\text {sat })}\right| \simeq$ $0.1 \mathrm{GV} / \mathrm{m}$ for $R_{\mathrm{tr}}<10 \mu \mathrm{m}$. 


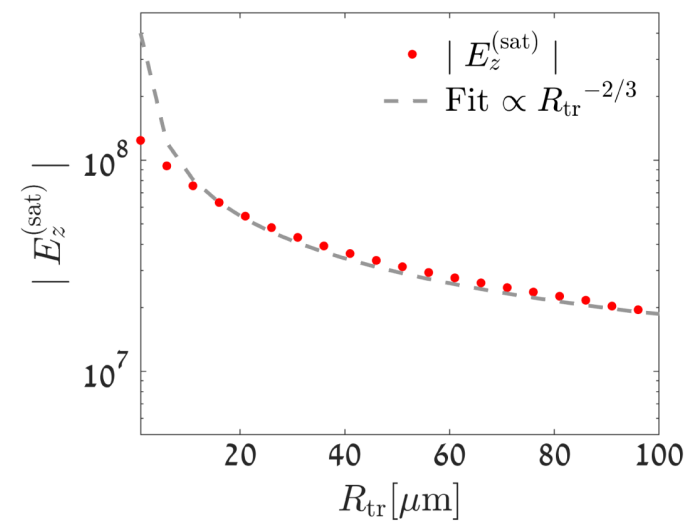

(a)

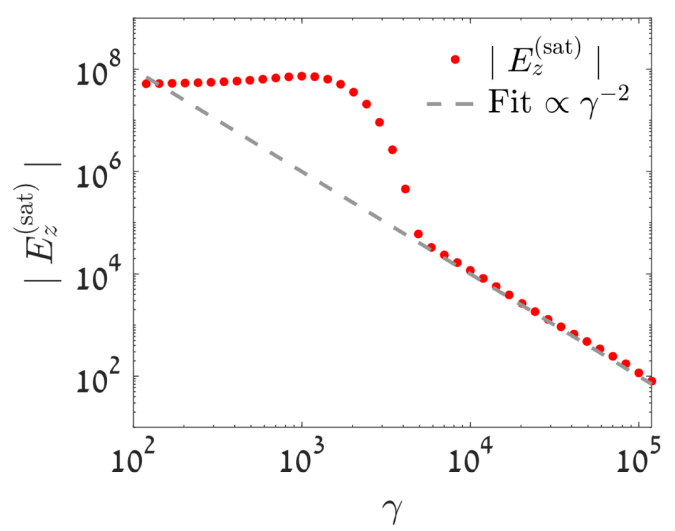

(b)

FIG. 19. Gradient at saturation as a function of the trigger bunch energy $\gamma$ (a) and trailing beam radius $R_{\mathrm{tg}}$ (b) where we take $h=R+R_{\mathrm{tg}}$ for $R=0.1 \mathrm{~mm}$.

Figure 19(b) shows the dependency on the gradient at saturation on the trigger beam energy $\gamma$. We find that for $\gamma<10^{3}$ the gradient is roughly constant, whereas for higher values there is a sharp decrease followed by a constant slope; this trend is similar for different geometries. To summarize, for the range $\gamma<10^{3}$, the maximal gradient at saturation is $\gamma$ independent

$$
\left|E_{z}^{(\text {sat,max })}\right| \simeq 50[\mathrm{MV} / \mathrm{m}]\left(\frac{50[\mu \mathrm{m}]}{R_{\mathrm{tr}}}\right)^{2 / 3}
$$

while for the higher energies where $\gamma \geq 3 \times 10^{3}$ the relation reads

$$
\left|E_{z}{ }^{(\mathrm{sat})}\right|_{\gamma \geq 3 \times 10^{3}} \simeq 0.1[\mathrm{MV} / \mathrm{m}]\left(\frac{50[\mu \mathrm{m}]}{R_{\mathrm{tr}}}\right)^{2 / 3}\left(\frac{3 \times 10^{3}}{\gamma}\right)^{2}
$$

\section{Summary}

In this section we conclude the analysis of a single rod configuration where the trigger electron bunch travels at a height $h$ parallel to the active medium surface. We find that the asymmetry of the structure results in multiple azimuthal harmonics, each with potentially multiple interacting modes. The number of modes for a single harmonic can be reduced to one for a design with $R \simeq 0.1 \mathrm{~mm}$, yet several harmonics have similar contributions. This presents a strong disadvantage as compared with the hollow rod configuration, which may facilitate a single dominant mode for the appropriate parameters.

We find that the gain parameter inducing the exponential growth is $\gamma$ independent, however the trigger field generated by the trigger electron bunch strongly depends on the electron energy $\gamma$ and the trailing beam radius as indicated in Eq. (C15). Moreover, we show that the maximal gradient at saturation for a few $\mu \mathrm{m}$ trailing beam radius is at the scale of $0.1 \mathrm{GV} / \mathrm{m}$ according to (C16), however this is an overestimation as the analysis relies on a single dominant harmonic which is not necessarily feasible. This value is an order of magnitude smaller than achievable for the hollow rod structure, which can reach gradients of approximately $1 \mathrm{GV} / \mathrm{m}$ with the proper design.

In conclusion, the single rod configuration presents several disadvantages which may impair its performance as an accelerating structure, as compared with the hollow rod configuration described in the main text. However, it will be instructive to examine the performance of this structure when employing ideal parameters of $R=0.1 \mathrm{~mm}, \gamma<10^{3}$ for the trigger bunch and $R_{\mathrm{tg}}<10 \mu \mathrm{m}$ which may facilitate an accelerating gradient of roughly $0.1 \mathrm{GV} / \mathrm{m}$.

[1] D. Greene and P. C. Williams, Linear Accelerators for Radiation Therapy (Taylor \& Francis Group, New York, NY, 1997).

[2] F. Zimmermann, Proc. Sci., 007 (2009).

[3] P. B. Wilson, Rev. Accel. Sci. Techol. 01, 7 (2008).

[4] E. Esarey, C. Schroeder, and W. Leemans, Rev. Mod. Phys. 81, 1229 (2009).

[5] C. Joshi and V. Malka, New J. Phys. 12, 045003 (2010).

[6] M. Aicheler, P. Burrows, M. Draper, T. Garvey, P. Lebrun, K. Peach, N. Phinney, H. Schmickler, D. Schulte, and N. Toge, A Multi-TeV linear collider based on CLIC technology: CLIC Conceptual Design Report, Tech. Rep. (SLAC National Accelerator Lab., Menlo Park, CA, 2014).

[7] W. Gai, P. Schoessow, B. Cole, R. Konecny, J. Norem, J. Rosenzweig, and J. Simpson, Phys. Rev. Lett. 61, 2756 (1988).

[8] M. Voin and L. Schächter, Phys. Rev. Accel. Beams 18, 071302 (2015).

[9] Z. Toroker, M. Voin, and L. Schächter, High Power Laser Sci. Eng. 2, e29 (2014).

[10] S. Banna, A. Mizrahi, and L. Schaechter, Laser Photonics Rev. 3, 97 (2009).

[11] Z. Toroker and L. Schächter, Phys. Rev. Accel. Beams 18, 071301 (2015). 
[12] W. Koechner, Solid-state Laser Engineering (Springer, New York, 2013), Vol. 1.

[13] A. Mizrahi and L. Schächter, Phys. Rev. E 70, 016505 (2004).

[14] X. E. Lin, Phys. Rev. Accel. Beams 4, 051301 (2001).

[15] K. J. Leedle, A. Ceballos, H. Deng, O. Solgaard, R. F. Pease, R. L. Byer, and J. S. Harris, Opt. Lett. 40, 4344 (2015).

[16] K.-Y. Ng, Phys. Rev. D 42, 1819 (1990).

[17] S. Shchelkunov, T. Marshall, G. Sotnikov, J. Hirshfield, W. Gai, M. Conde, J. Power, D. Mihalcea, and Z. Yusof, Phys. Rev. Accel. Beams 15, 031301 (2012).

[18] A. F. Oskooi, L. Zhang, Y. Avniel, and S. G. Johnson, Opt. Express 16, 11376 (2008).

[19] S. N. Galyamin, A. V. Tyukhtin, V. V. Vorobev, A. A. Grigoreva, and A. S. Aryshev, Phys. Rev. Accel. Beams 22, 012801 (2019).

[20] J. L. Hirshfield, Coaxial two-channel dielectric wakefield accelerator, Tech. Rep. (Omega-P, Inc., New Haven, CT, 2013).

[21] R. H. Pantell and H. E. Puthoff, Fundamentals of Quantum Electronics (John Wiley \& Sons, New York, 1969).
[22] R. Paschotta, J. Nilsson, A. C. Tropper, and D. C. Hanna, IEEE J. Quantum Electron. 33, 1049 (1997).

[23] D. Carlson, J. Appl. Phys. 39, 4369 (1968).

[24] P. Glas, M. Naumann, A. Schirrmacher, and T. Pertsch, Opt. Commun. 166, 71 (1999).

[25] B. Stuart, M. Feit, A. Rubenchik, B. Shore, and M. Perry, Phys. Rev. Lett. 74, 2248 (1995).

[26] A. Hanuka, K. Wootton, Z. Wu, K. Soong, I. Makasyuk, R. England, and L. Schächter, High Power Laser Sci. Eng. 7, e7 (2019).

[27] A. Hanuka and L. Schächter, Phys. Rev. Accel. Beams 21, 054001 (2018).

[28] A. Hanuka and L. Schächter, Phys. Rev. Accel. Beams 21, 064402 (2018).

[29] A. Hanuka and L. Schächter, Nucl. Instrum. Methods Phys. Res., Sect. A 888, 147 (2018).

[30] S. Banna, D. Schieber, and L. Schächter, J. Appl. Phys. 95, 4415 (2004).

[31] S. Iida and T. Uchiyama, Micro and Nano Engineering 3, 44 (2019). 